\title{
Neutron diffraction study of spin and charge ordering in $\mathrm{SrFeO}_{3-\delta}$
}

\author{
M. Reehuis, ${ }^{1,{ }^{*}}$ C. Ulrich, ${ }^{2,3,4}$ A. Maljuk, ${ }^{2,5}$ Ch. Niedermayer, ${ }^{6}$ B. Ouladdiaf, ${ }^{7}$ A. Hoser, ${ }^{1}$ T. Hofmann, ${ }^{1}$ and B. Keimer ${ }^{2, \dagger}$ \\ ${ }^{1}$ Helmholtz-Zentrum Berlin für Materialien und Energie, D-14109 Berlin, Germany \\ ${ }^{2}$ Max Planck Institute for Solid State Research, Heisenbergstrasse 1, D-70569 Stuttgart, Germany \\ ${ }^{3}$ University of New South Wales, School of Physics, Sydney, NSW 2052, Australia \\ ${ }^{4}$ Australian Nuclear Science and Technology Organisation (ANSTO), Locked Bag 2001, Kirrawee DC, NSW 2232, Australia \\ ${ }^{5}$ Leibniz Institut für Festkörper- und Werkstoffforschung (IFW) Dresden, Helmholtzstrasse 20, D-01171 Dresden, Germany \\ ${ }^{6}$ Labor für Neutronenstreuung, Paul-Scherrer-Institut, CH-5232 Villigen, Switzerland \\ ${ }^{7}$ Institut Laue-Langevin, Boîte Postale 156, F-38042 Grenoble Cedex 9, France
}

(Received 16 February 2012; published 22 May 2012)

\begin{abstract}
We report a comprehensive neutron diffraction study of the crystal structure and magnetic order in a series of single-crystal and powder samples of $\mathrm{SrFeO}_{3-\delta}$ in the vacancy range $0 \leqslant \delta \leqslant 0.23$. The data provide detailed insights into the interplay between the oxygen vacancy order and the magnetic structure of this system. In particular, a crystallographic analysis of data on $\mathrm{Sr}_{8} \mathrm{Fe}_{8} \mathrm{O}_{23}$ revealed a structural transition between the high-temperature tetragonal and a low-temperature monoclinic phase with a critical temperature $T=75 \mathrm{~K}$, which originates from charge ordering on the Fe sublattice and is associated with a metal-insulator transition. Our experiments also revealed a total of seven different magnetic structures of $\mathrm{SrFeO}_{3-\delta}$ in this range of $\delta$, only two of which (namely an incommensurate helix state in $\mathrm{SrFeO}_{3}$ and a commensurate, collinear antiferromagnetic state in $\mathrm{Sr}_{4} \mathrm{Fe}_{4} \mathrm{O}_{11}$ ) had been identified previously. We present a detailed refinement of some of the magnetic ordering patterns and discuss the relationship between the magnetotransport properties of $\mathrm{SrFeO}_{3-\delta}$ samples and their phase composition and magnetic microstructure.
\end{abstract}

DOI: 10.1103/PhysRevB.85.184109

PACS number(s): 61.05.fm, 61.66.Fn

\section{INTRODUCTION}

The physical properties of manganese oxides have been the focus of much research activity over the past two decades, with particular emphasis on the "colossal magnetoresistance" effect. The compound $\mathrm{SrFeO}_{3}$ crystallizes in a perovskite structure closely similar to the one of $\mathrm{LaMnO}_{3}$, the progenitor of one of the most extensively studied manganate families. Moreover, the $\mathrm{Fe}^{4+}$ ions in $\mathrm{SrFeO}_{3}$ exhibit the same high-spin $3 d^{4}$ electron configuration as the $\mathrm{Mn}^{3+}$ ions in $\mathrm{LaMnO}_{3}$. Despite these similarities, the electronic phase behavior of these two materials is completely different. While $\mathrm{LaMnO}_{3}$ is a Mott insulator and shows orbital order and commensurate, collinear antiferromagnetism, $\mathrm{SrFeO}_{3}$ is metallic and orbitally degenerate at all temperatures and exhibits a transition to incommensurate, helical magnetic order at $T_{\mathrm{N}}=134 \mathrm{~K} .{ }^{1}$ The profound difference between the electronic properties of both compounds is believed to originate from the higher degree of covalency of the metal-oxide bond in $\mathrm{SrFeO}_{3}$, but a detailed microscopic model picture has not yet been developed. This is in part due to the complex materials chemistry of $\mathrm{SrFeO}_{3-\delta}$, which can be prepared in stoichiometric form $(\delta \approx 0)$ only under high oxygen pressure. Samples with $\delta>0$ exhibit a mixture of structural phases with different ordering patterns of oxygen ions, which go along with different arrangements of iron ions in square-pyramidal $\mathrm{FeO}_{5}$ and octahedral $\mathrm{FeO}_{6}$ coordination. ${ }^{2-6}$ In addition to the stoichiometric end member $\mathrm{SrFeO}_{3}$, which crystallizes in the cubic perovskite structure (space group $P m \overline{3} m$ ), these include tetragonal $\mathrm{Sr}_{8} \mathrm{Fe}_{8} \mathrm{O}_{23}$ (space group I4/mmm), orthorhombic $\mathrm{Sr}_{4} \mathrm{Fe}_{4} \mathrm{O}_{11}$ (space group Cmmm), and orthorhombic, incommensurately modulated $\mathrm{Sr}_{2} \mathrm{Fe}_{2} \mathrm{O}_{5}$ [superspace group $\left.I 2 / m(0 \beta \gamma) 0 s\right] .^{6-9}$ The crystal structures of the former three compounds, which are subjects of the current article, are shown in Fig. 1.
Prior work has led to the identification of three separate magnetoresistance effects in $\mathrm{SrFeO}_{3-\delta}$ with $\delta \leqslant 0.25$ (Refs. 10-14), which can be traced to an additional magnetic transition below the Néel temperature in $\mathrm{SrFeO}_{3}$ (Refs. 11 and 12), to a charge-ordering transition in $\mathrm{Sr}_{8} \mathrm{Fe}_{8} \mathrm{O}_{23}$, (Refs. 11 and 12), and to magnetic disorder in $\mathrm{Sr}_{4} \mathrm{Fe}_{4} \mathrm{O}_{11}$ (Refs. 10-14), respectively. The coexistence between different oxygen vacancyordered phases in samples with $\delta \neq 0$ has so far confounded the quantitative description of these effects, because the superposition of different diffraction patterns in mixed-phase samples greatly complicates the crystallographic identification and analysis of the underlying spin and charge-ordering patterns. At the same time, real-space observations of oxygen vacancy order by electron microscopy are difficult, because these structures are very susceptible to electron beam damage. ${ }^{15}$

Here we present a neutron diffraction study designed to address this problem. By virtue of a detailed crystallographic analysis of single-crystal and powder samples of $\mathrm{SrFeO}_{3-\delta}$ with different concentrations of oxygen vacancies, we have arrived at a detailed experimental description of the spin and charge-ordering patterns in this family of compounds. Specifically, we report a comprehensive determination of the crystal structure of $\mathrm{Sr}_{8} \mathrm{Fe}_{8} \mathrm{O}_{23}$, which yields insight into the chargeordering pattern at low temperature. In addition, we pinpoint the origin of the transport anomaly in nominally stoichiometric crystals of cubic $\mathrm{SrFeO}_{3}$ as a hitherto unidentified commensurate magnetic phase, whose onset strongly affects the domain structure of the helical majority phase. In the range $0 \leqslant \delta \leqslant$ 0.25 , we have identified a total of seven magnetic phases, only two of which-namely the incommensurate helical phase in $\mathrm{SrFeO}_{3}$ and a commensurate collinear phase in $\mathrm{Sr}_{4} \mathrm{Fe}_{4} \mathrm{O}_{11}$ had been described before. ${ }^{1,8}$ Among the newly identified phases are commensurate and incommensurate magnetic 

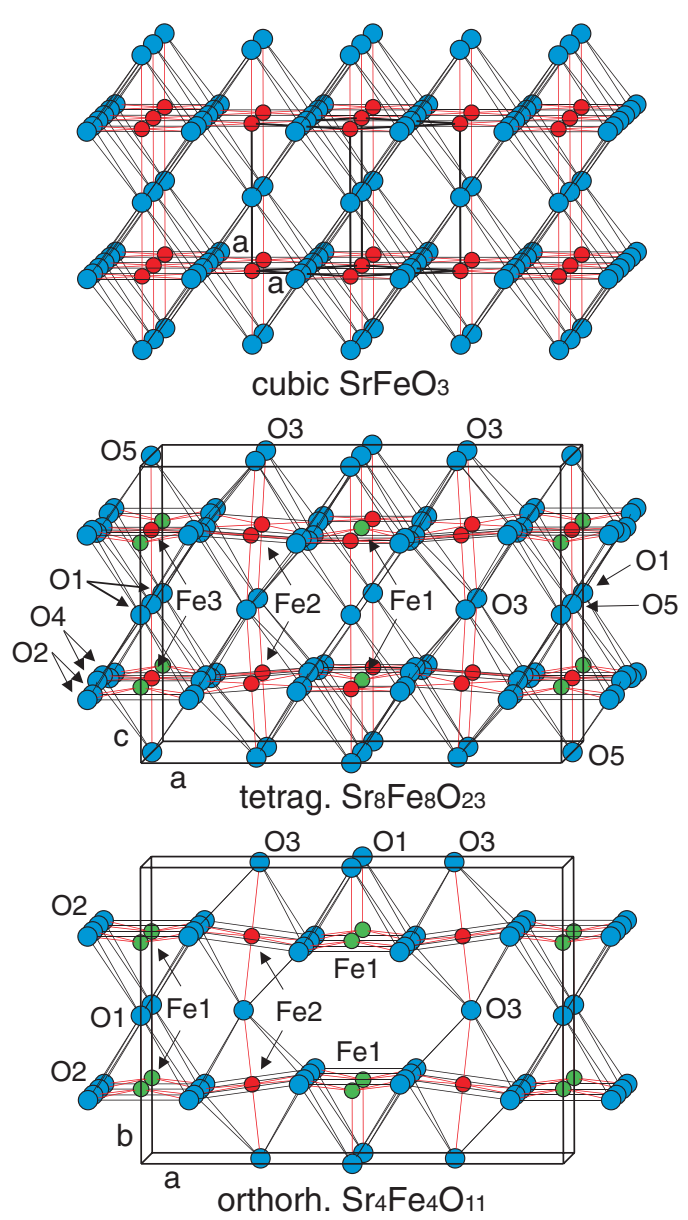

FIG. 1. (Color online) Crystal structures of vacancy-ordered ferrates in the system $\mathrm{SrFeO}_{3-x}$. For a clearer presentation, the $\mathrm{Sr}^{2+}$ ions are not shown. The network of cubic $\mathrm{SrFeO}_{3}$ is formed by undistorted corner-sharing $\mathrm{FeO}_{6}$ octahedra. In tetragonal ( $I$-centered) $\mathrm{Sr}_{8} \mathrm{Fe}_{8} \mathrm{O}_{23}$, every second $\mathrm{O}$ position along $[1,0,0]$ and $[0,1,0]$ (starting from the origin and the lattice point $\frac{1}{2}, \frac{1}{2}, \frac{1}{2}$ ) is unoccupied, so that sequences of alternating $\mathrm{FeO}_{6}$ octahedra and $\mathrm{FeO}_{5}$ square pyramids are generated. ( $\mathrm{Fe}$ atoms are marked in red and green). A further oxygen deficiency within these chains is found in orthorhombic $\mathrm{Sr}_{4} \mathrm{Fe}_{4} \mathrm{O}_{11}$, where one finds only corner-shared dimers of $\mathrm{FeO}_{5}$ square pyramids along [001].

structures in $\mathrm{Sr}_{8} \mathrm{Fe}_{8} \mathrm{O}_{23}$ as well as several weak magnetic structures that cannot be uniquely associated with any of the previously identified vacancy-ordered phases. This implies that the magnetic phase diagram of $\mathrm{SrFeO}_{3-\delta}$ is much richer than previously believed. The plethora of magnetic phases generated by oxygen vacancies in $\mathrm{SrFeO}_{3-\delta}$ is interesting in the context of recent work on magnetic field induced transitions and high-field magnetoresistance in $\mathrm{Sr}(\mathrm{Fe}, \mathrm{Co}) \mathrm{O}_{3}$ (Ref. 16), as well as related phenomena in other incommensurate magnets such as $\mathrm{BiFeO}_{3}$ (Ref. 17), $\mathrm{MnSi}$ (Ref. 18), and MnGe (Ref. 19).

\section{EXPERIMENTAL DETAILS}

High-quality oxygen-deficient single crystals of $\mathrm{SrFeO}_{3-\delta}$ with volumes of up to $0.25 \mathrm{~cm}^{3}$ were grown using the crucible free floating zone technique as described elsewhere. ${ }^{20}$
The oxygen deficiency depends on the growth conditions. Under a moderate oxygen pressure between 0.2 and 3.0 bars and a cooling rate of $20^{\circ} \mathrm{C}-30^{\circ} \mathrm{C} / \mathrm{h}$, an oxygen deficiency of $\delta=0.23 \pm 0.02$ was obtained. The oxygen content in single crystals was further increased $(0<\delta<0.09)$ under a postgrowth high-oxygen-pressure treatment at pressures up to 700 bars. ${ }^{21}$ In order to obtain the full stoichiometry of the end member $\mathrm{SrFeO}_{3}$, the single crystals were annealed at $5 \mathrm{kbar}$ oxygen pressure and a temperature of $400{ }^{\circ} \mathrm{C} .{ }^{21} \mathrm{In}$ addition, we have investigated powder samples of $\mathrm{SrFeO}_{3-\delta}$ with different oxygen contents. For the present study we annealed two powder samples at $400{ }^{\circ} \mathrm{C}$ in oxygen flow and in air, reaching oxygen deficiencies of $\delta=0.13 \pm 0.02$ and $\delta=$ $0.19 \pm 0.02$, respectively. The oxygen content in all samples was determined by thermogravimetry.

Single crystals of $\mathrm{SrFeO}_{3-\delta}(0 \leqslant \delta \leqslant 0.23)$ were investigated on the four-circle diffractometers E5 at the BER II reactor of the Helmholtz-Zentrum Berlin, and D10 at the Institut Laue-Langevin in Grenoble. Both instruments use pyrolytic-graphite $(\mathrm{PG})$ monochromators selecting the neutron wavelength $\lambda=2.36 \AA$ Additional neutron diffraction experiments were performed on the triple-axis instrument RITA II at the Swiss continuous neutron spallation source (SINQ) at the Paul-Scherrer Institut in Villigen, Switzerland, where the same neutron wavelength of $\lambda=2.36 \AA$ was used. This triple-axis instrument was used to measure selected Bragg reflections with higher accuracy and a significantly improved background. Complementary powder diffraction patterns of $\mathrm{SrFeO}_{3-\delta}$ with $\delta=0.13$ and $\delta=0.19$ were recorded on the powder diffractometer E6 at the BER II reactor of the HelmholtzZentrum Berlin, where the neutron wavelength $\lambda=2.44 \AA$ was selected by a PG monochromator. Additional powder patterns of $\mathrm{SrFeO}_{3-\delta}$ with $\delta=0.13$ were recorded on the high-intensity and high-resolution neutron powder diffractometers DMC and HRPT at the Paul-Scherrer-Institut. The instruments DMC and HRPT used a PG monochromator and a Ge monochromator selecting the neutron wavelengths $\lambda=2.57 \AA$ and $\lambda=1.158 \AA$, respectively. The refinements of the crystal and magnetic structure were carried out with the program FULLPROF (Ref. 22) with the nuclear scattering lengths $b(\mathrm{O})=5.805 \mathrm{fm}, b(\mathrm{Fe})=$ $9.54 \mathrm{fm}$, and $b(\mathrm{Sr})=7.02 \mathrm{fm} .{ }^{23}$ The magnetic form factors of the $\mathrm{Fe}^{3+}$ and $\mathrm{Fe}^{4+}$ ions were taken from Ref. 24 .

\section{RESULTS AND DISCUSSION}

\section{A. Coexistence of vacancy-ordered and magnetic phases in $\mathrm{SrFeO}_{3-\delta}$}

We begin our presentation by describing the determination of the structural and magnetic phase composition of our $\mathrm{SrFeO}_{3-\delta}$ samples. To this end, we measured the temperature dependence of various nuclear and magnetic Bragg reflections from single crystals with oxygen deficiencies of $\delta=0.0,0.03$, 0.13 , and 0.23 . Figure 1 shows that the iron sublattices in the crystal structures of $\mathrm{SrFeO}_{3}, \mathrm{Sr}_{8} \mathrm{Fe}_{8} \mathrm{O}_{23}$, and $\mathrm{Sr}_{4} \mathrm{Fe}_{4} \mathrm{O}_{11}$ are very similar. This implies that the strong nuclear reflections appear at almost the same $2 \theta$ positions, although the coordination of some fraction of the iron atoms changes from octahedral $\left(\mathrm{FeO}_{6}\right)$ to square pyramidal $\left(\mathrm{FeO}_{5}\right)$, and the associated lattice distortions lower the space-group symmetry. 

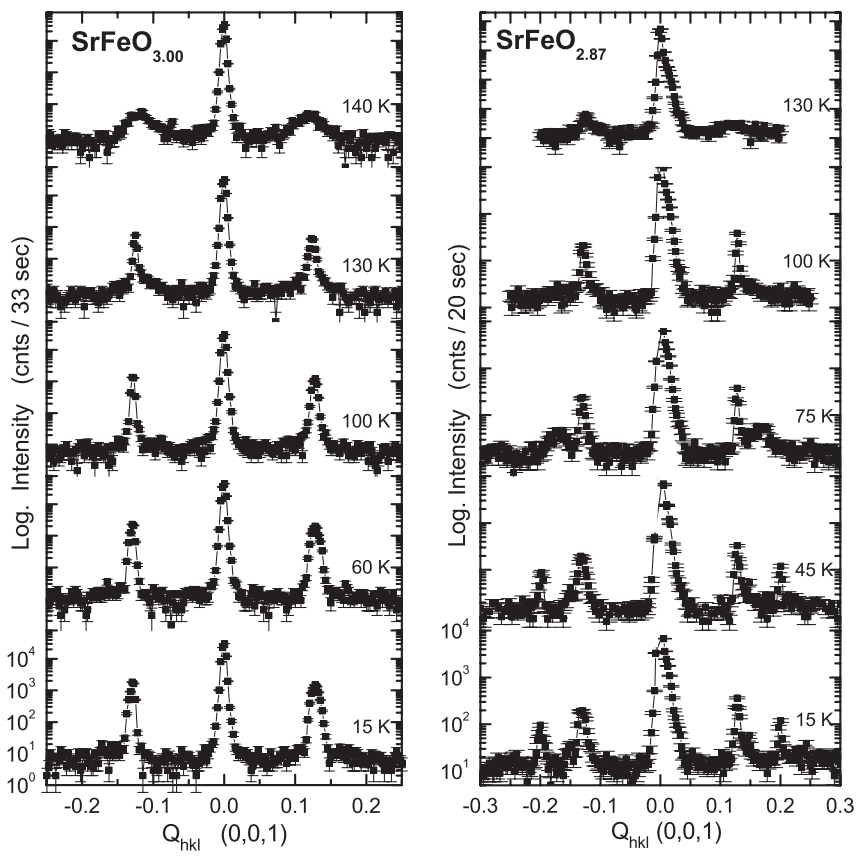

FIG. 2. Neutron diffraction data on the single crystals of composition $\mathrm{SrFeO}_{3.00}$ and $\mathrm{SrFeO}_{2.87}$ measured at various temperatures. The scans were performed along the $[1,1,1]$ direction around the structural Bragg reflection $(0,0,1)_{\text {cub. With decreasing temperature }}$ magnetic reflections of phase I appeared in both samples. In the oxygen-deficient crystal $\mathrm{SrFeO}_{2.87}$ an additional set of magnetic reflection of phase II could be observed.

Therefore, we give the indices of the measured reflections $(h, k, \ell)$ in the cubic setting, in addition to the tetragonal and/or orthorhombic settings that characterize the full lattice symmetry of the oxygen-deficient compounds.

Figure 2 shows typical neutron diffraction data on the $\mathrm{SrFeO}_{3.00}$ and $\mathrm{SrFeO}_{2.87}$ single crystals. The measurements were performed at various temperatures along the $[1,1,1]$ direction around the structural Bragg reflection $(0,0,1)_{\text {cub. }}$. We first discuss the data on the crystal with the ideal composition $\mathrm{SrFeO}_{3.00}$, which served as the calibration standard for the determination of the phase fractions of the other samples. The $\mathrm{SrFeO}_{3.00}$ crystal exhibits only one set of magnetic satellite peaks at the incommensurate wave vector $(\Delta, \Delta, \Delta)$ with $\Delta=$ 0.129 at low temperatures. This signal can be attributed to the previously identified helical order of the iron moments (termed "phase I" in the present work) in the cubic structure of $\mathrm{SrFeO}_{3} .{ }^{1}$ The temperature dependence of the corresponding magnetic Bragg peak intensity is displayed in Fig. 3. The Bragg reflections of phase I vanish upon heating above the Néel temperature $T_{\mathrm{N}}=133(1) \mathrm{K}$, which is in excellent agreement with $T_{\mathrm{N}}=134 \mathrm{~K}$ given earlier. ${ }^{1}$

The diffraction data of the $\mathrm{SrFeO}_{2.87}$ crystal, also presented in Fig. 2, are characteristic of mixed-phase samples. In addition to the phase-I reflections, this sample exhibits a second set of magnetic reflections with $\Delta=0.20$, which disappear for temperatures above $T_{\mathrm{S}}=75(2) \mathrm{K}$. The onset temperature of the magnetic structure indicated by these reflections (henceforth termed "phase II") coincides with the onset of magnetic and charge order previously observed by Mössbauer spectroscopy, transport, optical, and Raman scattering experiments in the tetragonal ferrate $\mathrm{Sr}_{8} \mathrm{Fe}_{8} \mathrm{O}_{23}$ (Refs. 11 and 12). The presence of a structural phase transition due to charge ordering is confirmed by an intensity change of the nuclear reflection $(2,0,0)_{\text {cub }}\left[(4,4,0)_{\text {tetr }} /(0,0,4)_{\text {tetr }}\right]$ at $T_{\mathrm{S}}$ (Fig. 4). The main cause of the intensity anomalies of the main nuclear Bragg reflections at the charge-ordering transition is a modification of the mosaic character of the single crystals due to lattice strains and a consequent change in the extinction of the neutron beam. A detailed crystallographic analysis (see Sec. III B) indicates a small additional contribution from atomic displacements associated with charge ordering.

Figure 4 also displays corresponding data for the crystal of composition $\mathrm{SrFeO}_{2.97}$, where the anomaly of the $(2,0,0)_{\text {cub }}$ reflection is reduced compared to the one in the $\mathrm{SrFeO}_{2.87}$ crystal, and the intensity of the magnetic satellite at $(0.129$, $0.871,0.871)_{\text {cub }}$ is correspondingly enhanced. The transition temperatures ( $T_{\mathrm{S}}=75$ and $T_{\mathrm{N}}=133 \mathrm{~K}$, respectively), on the other hand, do not depend on oxygen content. This indicates that these crystals contain different fractions of the cubic $\left(\mathrm{SrFeO}_{3}\right)$ and vacancy-ordered tetragonal $\left(\mathrm{Sr}_{8} \mathrm{Fe}_{8} \mathrm{O}_{23}\right)$ phases. Twinning of the crystal structures of the lower-symmetric ferrates and the presence of various minority phases (see below) greatly complicate the full refinement of the lattice and magnetic structures in the single crystals with $\delta>0$. In order to estimate the phase composition of these samples, we have therefore used the helical magnetic structure of $\mathrm{SrFeO}_{3}$, whose ordered moment $\left[\mu_{\exp }=2.960(12) \mu_{\mathrm{B}}\right.$ per $\mathrm{Fe}^{4+}$ ion $]$ has been precisely determined at $2 \mathrm{~K}$ (see Sec. IIIC 1 ), in order to determine the overall scale factor of $\mathrm{SrFeO}_{3}$ from the intensity of the incommensurate magnetic reflection $(0.129$, $0.871,0.871)_{\text {cub. }}$ In this way, we found that the two samples $\mathrm{SrFeO}_{2.97}$ and $\mathrm{SrFeO}_{2.87}$ contain $74(2) \%$ and $26(2) \%$ of the cubic phase $\mathrm{SrFeO}_{3}$, respectively. The other main component in these samples is the tetragonal phase $\mathrm{Sr}_{8} \mathrm{Fe}_{8} \mathrm{O}_{23}$.

The oxygen content of the $\mathrm{SrFeO}_{2.77}$ crystal is close to that of the orthorhombic phase $\mathrm{Sr}_{4} \mathrm{Fe}_{4} \mathrm{O}_{11}\left(\mathrm{SrFeO}_{2.75}\right)$. In fact, magnetic intensity could be found on the position of the magnetic reflection $(1,1,1)_{\text {orth }}$, suggesting the presence of the previously identified commensurate magnetic structure of $\mathrm{Sr}_{4} \mathrm{Fe}_{4} \mathrm{O}_{11}$ (here termed "phase VII"). Figure 5 shows that the magnetic intensity vanishes at the Néel temperature $T_{\mathrm{N}}=$ 232(2) K, which is in excellent agreement with the transition temperature given earlier. ${ }^{8}$

A comprehensive survey of the reciprocal lattice of our single-crystal samples revealed several sets of weaker magnetic reflections, which could not be uniquely associated with one of the structures displayed in Fig. 1 and may hence indicate small fractions of hitherto unidentified oxygen vacancy-ordered structures. In the crystals with composition $\mathrm{SrFeO}_{3.00}, \mathrm{SrFeO}_{2.87}$, and $\mathrm{SrFeO}_{2.77}$, we observed magnetic reflections that could be indexed as $\left(\frac{1}{4}, 0,0\right)_{\text {cub }}$ and $\left(\frac{3}{4}\right.$, $0,0)_{\text {cub }}$, or $\left(0,0, \frac{1}{2}\right)_{\text {tetr }}$ and $\left(0,0,1 \frac{1}{2}\right)_{\text {tetr }}$ in the tetragonal setting, below $T_{\mathrm{N}}=65(4) \mathrm{K}$ (Figs. 3 and 5). Their intensity in the $\mathrm{SrFeO}_{3.00}$ crystal is much weaker than in the other crystals, and may be due to residual oxygen defects below the detection level of 0.02 for $\delta$ in $\mathrm{SrFeO}_{3-\delta}$. The onset temperature of these reflections coincides with anomalies in Mössbauer and transport measurements previously reported in nominally stoichiometric $\mathrm{SrFeO}_{3-\delta}$ crystals. ${ }^{12}$ They can be 

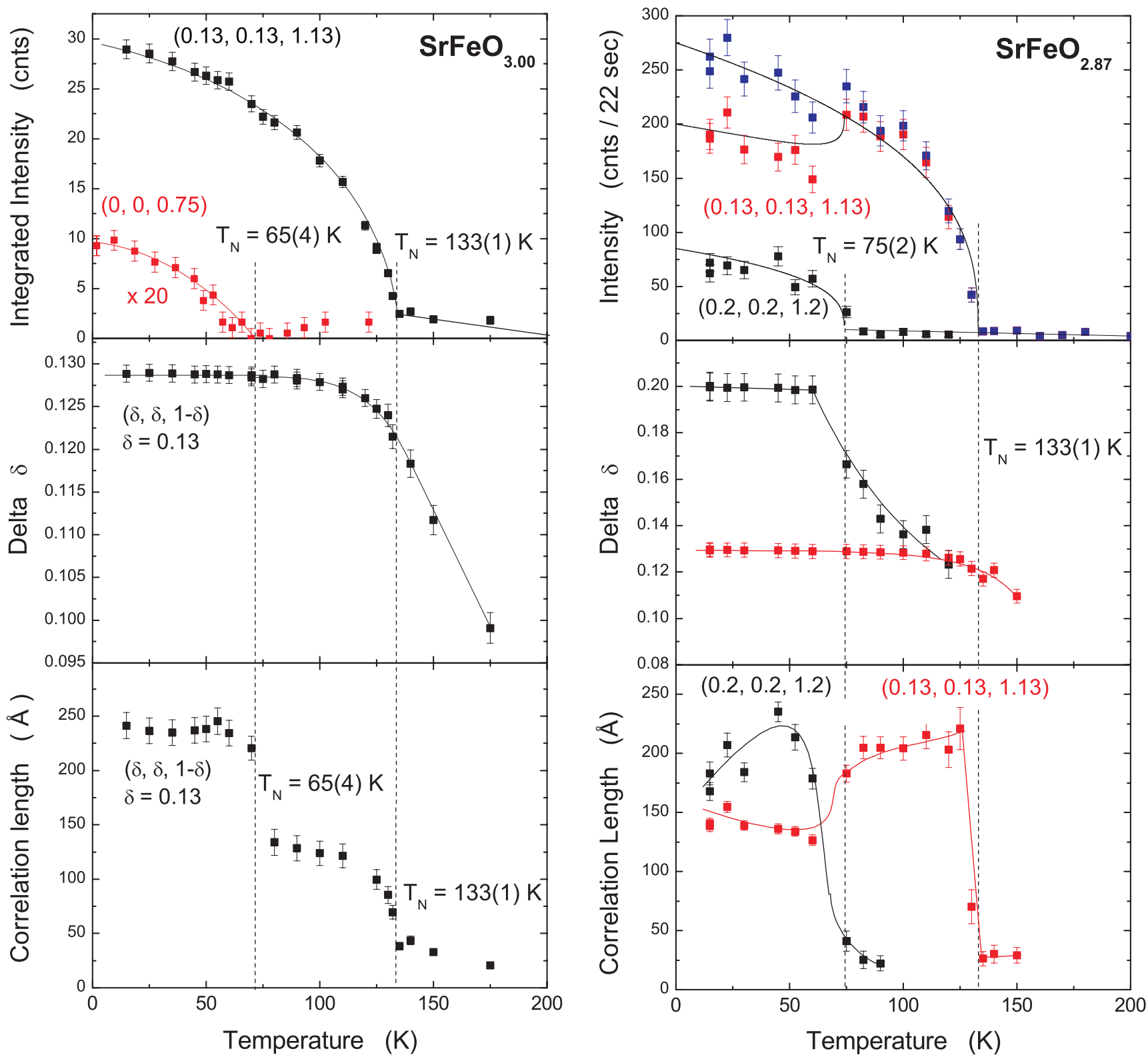

FIG. 3. (Color online) Temperature dependence of the intensity, incommensurability, and magnetic correlation length of the helical magnetic Bragg reflections of the magnetic phase $\mathrm{I}$ in the single crystal $\mathrm{SrFeO}_{3.00}$ (left) and the magnetic phases $\mathrm{I}$ and $\mathrm{II}$ in $\mathrm{SrFeO}{ }_{2.87}$ (right). For the sample $\mathrm{SrFeO}_{3.00}$ very weak intensity was found on the position $\left(0,0, \frac{3}{4}\right)_{\text {cub }}$ indicating the presence of an additional magnetic phase (denoted as phase IV in the text) for temperatures below 65(4) K. The solid lines in the upper panels are the results of power-law fits to the measured intensity. It is interesting to note that the incommensurability is temperature dependent close to the magnetic phase transition.

generated with the propagation vector $\boldsymbol{k}=\left(0,0, \frac{1}{2}\right)$, indicating a magnetic unit cell where the tetragonal $c$ axis is doubled. We refer to this structure as "phase IV" and discuss its properties in detail in Sec. III C 4.

In the $\mathrm{SrFeO}_{2.77}$ crystal, very weak magnetic intensity could be observed at the position $(0.30,0.30,0.75)_{\text {cub }}$. The peak intensity disappears upon heating above $T=110(4)$ $\mathrm{K}$, which coincides with anomalies in susceptibility and resistivity measurements on samples with different oxygen contents (Fig. 5). ${ }^{12}$ Furthermore, incommensurate magnetic Bragg reflections were observed at $(0.79,0.79,0)_{\text {cub }}$ and $(1.21,1.21,0)_{\text {cub }}$ which disappear at a transition temperature of $T=60(5) \mathrm{K}$. The incommensurate magnetic phases $\mathrm{V}$ and VI indicated by these two sets of reflections may be attributed to small volumes of hitherto unidentified oxygendeficient ferrates with crystal structures very similar to those of $\mathrm{Sr}_{8} \mathrm{Fe}_{8} \mathrm{O}_{23}$ and $\mathrm{Sr}_{4} \mathrm{Fe}_{4} \mathrm{O}_{11}$, which are intergrown in the majority-phase matrix. Due to the weakness of the observed reflections, the magnetic structures of phases V and VI could not be determined from the available data. However, a description of these structures in the tetragonal setting implies that incommensurate ordering occurs in the basal $a b$ plane.

The widths of the magnetic reflections are generally significantly larger than the instrumental resolution and exhibit 


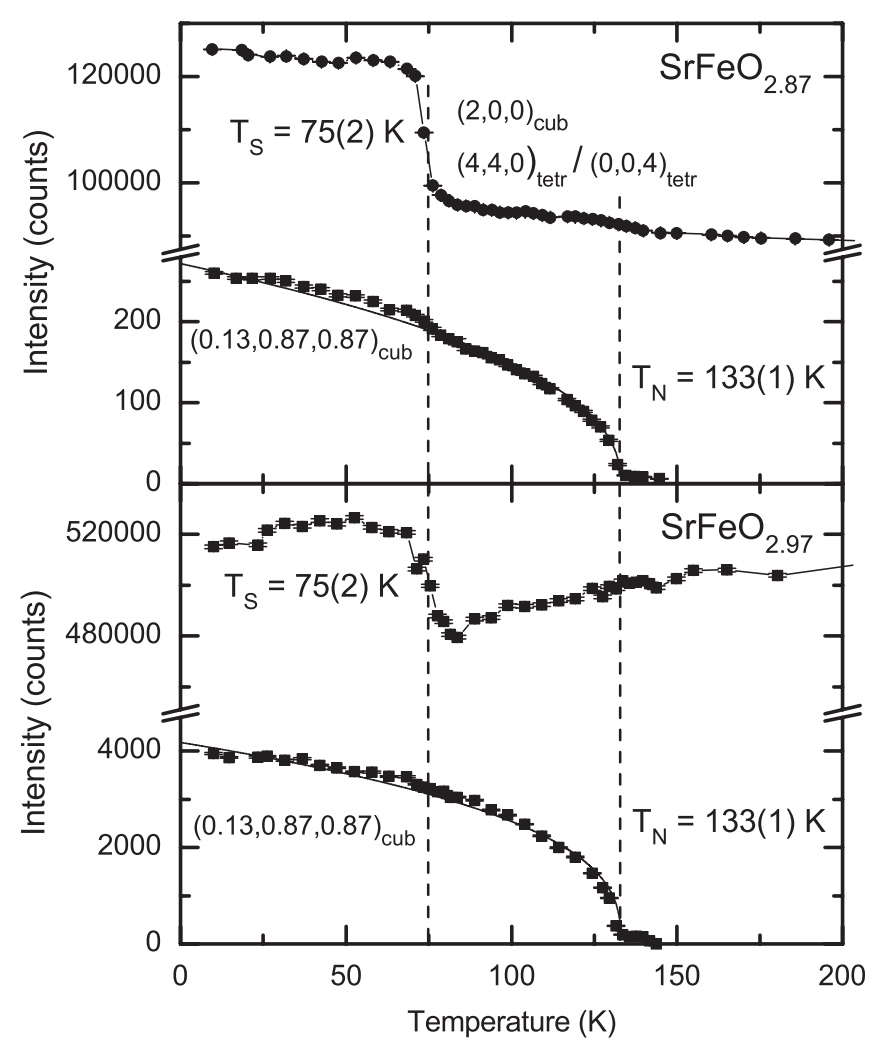

FIG. 4. Temperature dependence of the strong nuclear reflection $(200)_{\text {cub }}$ of $\mathrm{SrFeO}_{3-\delta}$ and the magnetic reflection $(0.13,0.87,0.87)_{\text {cub }}$ of the cubic phase $\mathrm{SrFeO}_{3}$ (denoted as phase I). For the samples with lower oxygen content, the reflection $(2,0,0)_{\text {cub }}\left[(4,4,0)_{\text {tetr }} /(0,0\right.$, $4)_{\text {tetr }}$ ] shows a strong change of intensity at $T_{\mathrm{S}}=75(2) \mathrm{K}$ indicating the presence of a structural phase transition. This transition is more pronounced in the sample $\mathrm{SrFeO}_{2.87}$, suggesting that this transition occurs in the tetragonal phase $\mathrm{Sr}_{8} \mathrm{Fe}_{8} \mathrm{O}_{23}$.

interesting temperature dependences (Fig. 3). In the $\mathrm{SrFeO}_{3.00}$ crystal, the widths of the incommensurate phase-I reflections at high temperatures indicate a magnetic domain size of $\sim 100 \AA$ (Fig. 3, left panel). The small domain size probably reflects phason disorder, along with a distribution of different polarization domains. Below the onset temperature of phase IV, however, the domain size inferred from the width of the phase-I reflections increases by a factor of 2 , possibly because the magnetic order in the phase-IV domains locks in the phase relationship between some of the phase-I domains. The strong influence of the onset of phase IV on the microstructure of phase I, despite the small volume fraction of phase IV, implies that both phases are not macroscopically separated, but rather densely intertwined. A possible scenario is that the small number of residual oxygen vacancies that generate phase IV form extended planar structures, as they do in $\mathrm{Sr}_{8} \mathrm{Fe}_{8} \mathrm{O}_{23}$ and $\mathrm{Sr}_{4} \mathrm{Fe}_{4} \mathrm{O}_{11}$, but with a different periodicity.

A related effect is observed in the $\mathrm{SrFeO}_{2.87}$ crystal, where the size of the phase-I domains decreases below the onset temperature of phase II (Fig. 3, right panel). At the same temperature, the growth of the phase-I intensity upon cooling is arrested, possibly because some of the volume occupied by phase I for $T>T_{\mathrm{S}}=75 \mathrm{~K}$ is converted to phase II. These data indicate that the interplay of the oxygen vacancy order and the

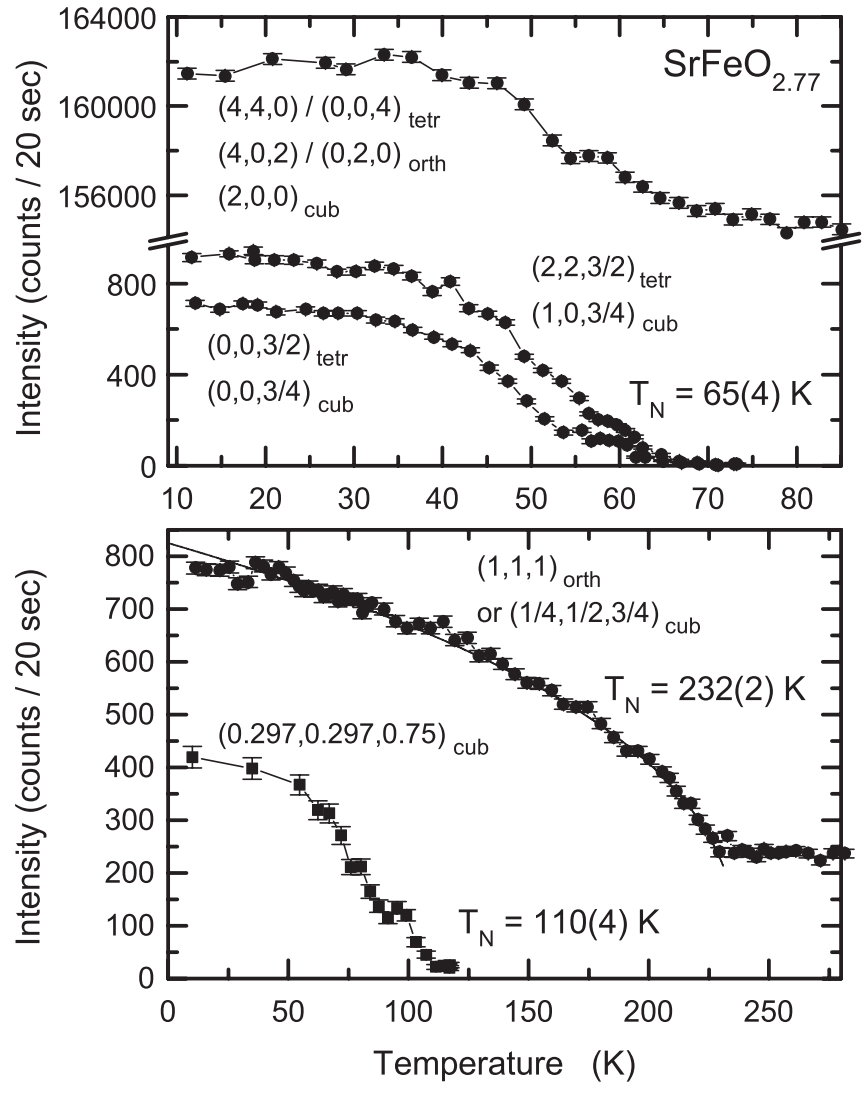

FIG. 5. Temperature dependence of the reflections $(2,0,0)_{\text {cub }},(0$, $\left.0, \frac{3}{4}\right)_{\text {cub }},\left(1,0, \frac{3}{4}\right)_{\text {cub }}$, and $\left(\frac{1}{4}, \frac{1}{2}, \frac{3}{4}\right)_{\text {cub }}$ of $\mathrm{SrFeO}_{2.77}$. Magnetic intensity of the $\left(\frac{1}{4}, \frac{1}{2}, \frac{3}{4}\right)_{\text {cub }}\left[(1,1,1)_{\text {orth }}\right.$ in the orthorhombic setting $]$ appears spontaneously at 232(2) $\mathrm{K}$ due to the onset of antiferromagnetic ordering of the $\mathrm{Fe}$ atoms (phase VII) in orthorhombic $\mathrm{Sr}_{4} \mathrm{Fe}_{4} \mathrm{O}_{11}$. A second magnetic transition was observed at $T_{\mathrm{N}}=65(3) \mathrm{K}$. This can be ascribed to an antiferromagnetic ordering in a second phase, which could not be characterized so far. Furthermore, magnetic Bragg reflections with $T_{\mathrm{N}}=110(4) \mathrm{K}$ were observed at the incommensurate positions ( $h \pm 0.297, k \pm 0.297, \ell \pm 0.25)_{\text {cub }}$ (phase $\mathrm{V}$ ).

magnetic structure generates a complex, nanoscopic magnetic landscape.

Complementary neutron diffraction experiments were performed on $\mathrm{SrFeO}_{3-\delta}$ powder samples with oxygen deficiencies $\delta=0.13$ and $\delta=0.19$, in the temperature range between 10 and $240 \mathrm{~K}$. The experiments were carried out on the instruments E6 and DMC using the larger neutron wavelengths $\lambda=2.44 \AA$ and $\lambda=2.57 \AA$, respectively. The powder patterns of the sample with $\delta=0.13$, presented in Fig. 6 , clearly show the appearance of individual sets of magnetic reflections with different ordering temperatures. The strongest magnetic reflection, observed at $2 \theta=8.3^{\circ}$, could be identified as the magnetic satellite $(0.128,0.128,0.128)_{\text {cub }}$ of the helical phase I, which appears below the Néel temperature $T_{\mathrm{N}}=133(1) \mathrm{K}$. The wave vector components were found to be closely similar to those obtained from our single-crystal diffraction study. A second set of four incommensurate magnetic reflections appears below $T_{\mathrm{S}}=75(2) \mathrm{K}$, the charge-ordering temperature of $\mathrm{Sr}_{8} \mathrm{Fe}_{8} \mathrm{O}_{23}$ already discussed above. However, the magnetic structure indicated by these reflections is different from the one 


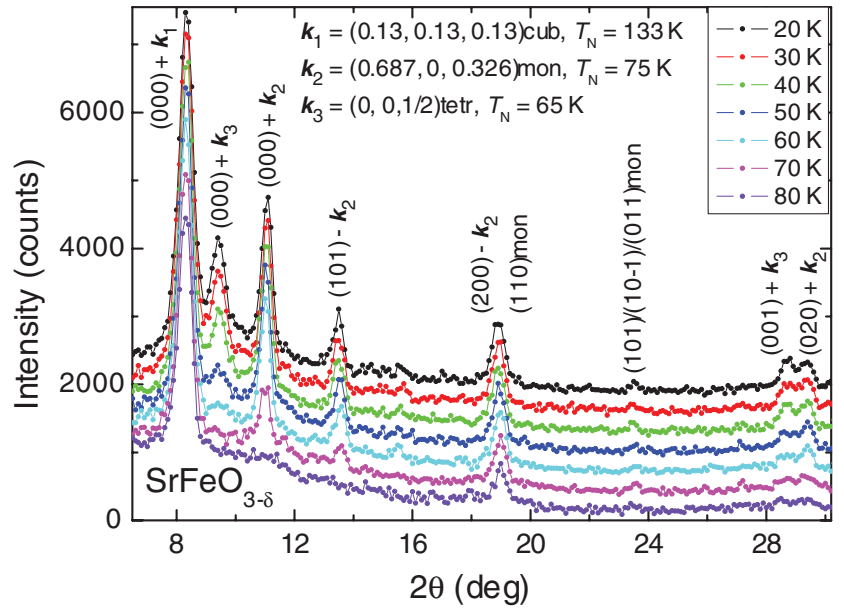

FIG. 6. (Color online) Neutron powder patterns of $\mathrm{SrFeO}_{3-\delta}$ with $\delta=0.13$ at different temperatures between 20 and $80 \mathrm{~K}$. The traces for temperatures below $80 \mathrm{~K}$ have been shifted for clarity. The neutron wavelength was $\lambda=2.58 \AA$. Below $T_{\mathrm{N}}=133(1) \mathrm{K}$ magnetic intensity appears at the position $(0.13,0.13,0.13)_{\text {cub }}$ due to the incommensurate ordering (phase I) of the end member $\mathrm{SrFeO}_{3}$. Below $T_{\mathrm{N}}=75(2)$ $\mathrm{K}$ incommensurate magnetic order of the iron moments (phase III) with $\boldsymbol{k}_{2}=(0.687,0,0.326)_{\text {mon }}$ (using the monoclinic unit cell of low-temperature structure of $\mathrm{Sr}_{8} \mathrm{Fe}_{8} \mathrm{O}_{23}$ ). Further commensurate magnetic reflections are observable below $T_{\mathrm{N}}=65(4) \mathrm{K}$, suggesting the presence of a third magnetic phase (phase IV), with $\boldsymbol{k}_{3}=$ $\left(0,0, \frac{1}{2}\right)_{\text {tetr }}$, of another oxygen-deficient ferrate, which could not be characterized so far.

identified in the same temperature range in the crystal with the same composition, $\mathrm{SrFeO}_{2.87}$. We therefore refer to this structure as "phase III". (Note that reflections characteristic of phase III were also found in the single-crystalline sample, albeit with much weaker intensity.) The strongest phase-III satellite, observed at $2 \theta=11.2^{\circ}$, could be indexed as $(0.169$, $0.169,0.169)_{\text {cub }}$ using the cubic setting. In contrast, the other three satellites of this set observed at $2 \theta$ angles of $13.6^{\circ}, 18.9^{\circ}$, and $29.6^{\circ}$ (Fig. 6) could not be generated by the rule $(h k \ell)_{\mathrm{M}}=$ $(h k \ell)_{\mathrm{N}} \pm \boldsymbol{k}$ using the cubic unit cell. Taking into account the much larger unit cell of the tetragonal phase $\mathrm{Sr}_{8} \mathrm{Fe}_{8} \mathrm{O}_{23}$ (cell dimensions $2 \sqrt{2} a \times 2 \sqrt{2} a \times 2 c$ referenced to the cubic cell), all of these satellites could be indexed. Here the cubic cell has been transformed to the tetragonal unit cell via the matrix (220; 2-20; 002). Consequently the propagation vector $\boldsymbol{k}=$ $(0.169,0.169,0.169)_{\text {cub }}$ changes to $\boldsymbol{k}=(0.676,0,0.338)_{\text {tetr }}$, where the $\boldsymbol{k}$ vector now lies in the tetragonal ac plane. Finally, magnetic reflections indicative of phase IV were detected at $2 \theta=9.5^{\circ}$ and $2 \theta=28.9^{\circ}$ (Fig. 6) below 65(4) K. In the cubic setting these two reflections could be indexed as $\left(\frac{1}{4}, 0,0\right)_{\text {cub }}$ and $\left(\frac{3}{4}, 0,0\right)_{\text {cub }}$, $\left[\right.$ or $\left(0,0, \frac{1}{2}\right)_{\text {tetr }}$ and $\left.\left(0,0,1 \frac{1}{2}\right)_{\text {tetr }}\right]$, as discussed above for the single crystal data.

In the powder sample with oxygen deficiency $\delta=0.19$, the cubic ferrate $\mathrm{SrFeO}_{3}$ was found to be absent, but magnetic reflections characteristic of the orthorhombic ferrate $\mathrm{Sr}_{4} \mathrm{Fe}_{4} \mathrm{O}_{11}$ were observed. With the knowledge of the magnetic structures of the different ferrates, we were able to determine the composition of the two powder samples (see Sec. III C 4).

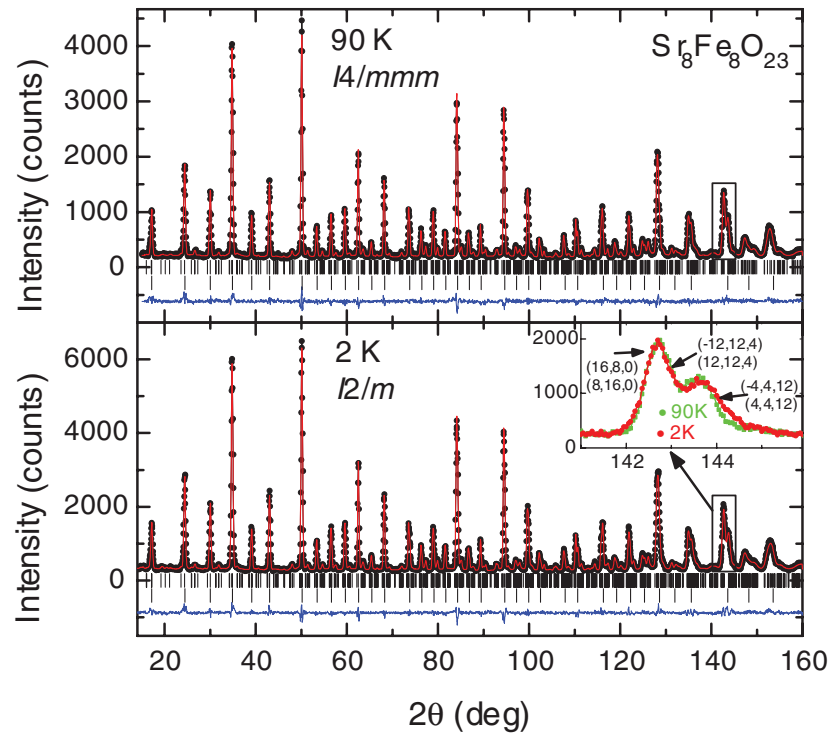

FIG. 7. (Color online) Neutron powder patterns of $\mathrm{Sr}_{8} \mathrm{Fe}_{8} \mathrm{O}_{23}$. From the data sets collected at 2 and $90 \mathrm{~K}$ the crystal structure was refined in the monoclinic and tetragonal space groups $I 2 / \mathrm{m}$ and $I 4 / \mathrm{mmm}$, respectively. The calculated patterns (red) are compared with the observed ones (black circles). The difference patterns (blue) as well as the peak positions (black bars) of $\mathrm{Sr}_{8} \mathrm{Fe}_{8} \mathrm{O}_{23}$ (above) and the cubic phase $\mathrm{SrFeO}_{3}$ (below) are given in the lower part of each diagram. The inset indicates that the $2 \theta$ positions of reflections $(h$, $k, \ell$ ) with large $\ell$ showed the strongest changes below the structural phase transition.

\section{B. Crystal structure and charge-ordering transition of $\mathrm{Sr}_{8} \mathrm{Fe}_{8} \mathrm{O}_{23}$}

The data presented in the previous section demonstrate that all of the oxygen-deficient single-crystalline samples contained at least three different vacancy-ordered structures. The lower-symmetric structures show twinning, and the tetragonal ferrate $\mathrm{Sr}_{8} \mathrm{Fe}_{8} \mathrm{O}_{23}$ exhibits additional twinning below the low-temperature structural phase transition associated with charge ordering. Therefore it was not possible to refine the crystal structures of the oxygen-deficient ferrates from the single-crystal diffraction data. In order to investigate the crystal structure of $\mathrm{Sr}_{8} \mathrm{Fe}_{8} \mathrm{O}_{23}\left(\mathrm{SrFeO}_{2.875}\right)$ we thus collected highresolution neutron powder patterns (Fig. 7) using a sample with composition $\mathrm{SrFeO}_{2.87}$ which mostly contains $\mathrm{Sr}_{8} \mathrm{Fe}_{8} \mathrm{O}_{23}$. The choice of the shorter neutron wavelength $\lambda=1.158 \AA$ allowed us to collect a relatively large number of nuclear Bragg reflections up to $\sin \theta / \lambda=0.85 \AA^{-1}$. Rietveld refinements of the powder data collected at $199 \mathrm{~K}$ in the tetragonal space group $I 4 / \mathrm{mmm}$ (No. 139) resulted in a residual $R_{F}=0.038$ (defined as $R_{F}=\sum\left\|F_{\text {obs }}|-| F_{\text {calc }}\right\| / \sum\left|F_{\text {obs }}\right|$ ) and a reduced $\chi^{2}=1.45$. The crystal structure of the minor phase $\mathrm{SrFeO}_{3}$ was refined simultaneously. In agreement with Hodges $e t$ al. (Ref. 6) we could not obtain a better fit using the orthorhombic and monoclinic space groups Fmmm $\left(R_{F}=0.055, \chi^{2}=\right.$ 2.01) and $I 2 / \mathrm{m}$ (setting: $I 112 / \mathrm{m})\left(R_{F}=0.058, \chi^{2}=\right.$ 2.05 ), respectively. The $\mathrm{Sr}, \mathrm{Fe}$, and $\mathrm{O}$ atoms in $I 4 / \mathrm{mmm}$ are located at the following Wyckoff positions: $\operatorname{Sr} 1$ in $8 i(x, 0,0)$, $\mathrm{Sr} 2$ in $8 j\left(x, 0, \frac{1}{2}\right), \mathrm{Fe} 1$ in $4 e(0,0, z), \mathrm{Fe} 2$ in $8 f\left(\frac{1}{4}, \frac{1}{4}, \frac{1}{4}\right), \mathrm{Fe} 3$ in 
TABLE I. Results of the crystal structure refinements of $\mathrm{Sr}_{8} \mathrm{Fe}_{8} \mathrm{O}_{23}$ from neutron powder diffraction $(\lambda=1.158 \AA)$. The refinements of the data sets collected at 90 and $199 \mathrm{~K}$ were carried out in the tetragonal space group $I 4 / \mathrm{mmm}$. For the data collected at $2 \mathrm{~K}$ the structure was

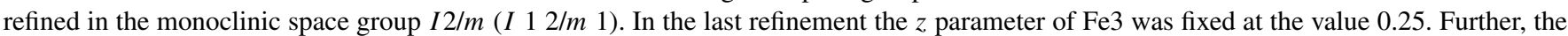
parameters $x$ and $y$ of $\mathrm{O} 3$ as well as the thermal parameters $B_{\text {is }}\left(\right.$ in $\left.\AA^{2}\right)$ of each element $\mathrm{Sr}, \mathrm{Fe}$, and $\mathrm{O}$ were constrained to be equal.

\begin{tabular}{|c|c|c|c|c|c|c|c|c|c|c|c|c|}
\hline & \multicolumn{4}{|c|}{$\mathrm{Sr}_{8} \mathrm{Fe}_{8} \mathrm{O}_{23}$ at $2 \mathrm{~K}$, in $\mathrm{I} 2 / \mathrm{m}$} & \multicolumn{4}{|c|}{$\mathrm{Sr}_{8} \mathrm{Fe}_{8} \mathrm{O}_{23}$ at $90 \mathrm{~K}$, in $\mathrm{I} / \mathrm{mmm}$} & \multicolumn{4}{|c|}{$\mathrm{Sr}_{8} \mathrm{Fe}_{8} \mathrm{O}_{23}$ at $199 \mathrm{~K}$, in $I 4 / \mathrm{mmm}$} \\
\hline & $x$ & $y$ & $z$ & $B_{\text {is }}$ & $x$ & $y$ & $z$ & $B_{\text {is }}$ & $x$ & $y$ & $z$ & $B_{\text {is }}$ \\
\hline $\operatorname{Sr} 1(1)$ & $0.2568(14)$ & 0 & $0.9968(19)$ & $0.07(2)$ & $0.2580(4)$ & 0 & 0 & $0.14(1)$ & $0.2586(4)$ & 0 & 0 & $0.25(1)$ \\
\hline $\operatorname{Sr} 1(2)$ & 0 & $0.2606(15)$ & 0 & 0.07 & - & - & - & 0.14 & - & - & - & 0.25 \\
\hline $\operatorname{Sr} 2(1)$ & $0.2462(13)$ & 0 & $0.5037(20)$ & 0.07 & $0.2519(4)$ & 0 & $\frac{1}{2}$ & 0.14 & $0.2536(3)$ & 0 & $\frac{1}{2}$ & 0.25 \\
\hline $\operatorname{Sr} 2(2)$ & 0 & $0.2532(12)$ & $\frac{1}{2}$ & 0.07 & - & - & - & 0.14 & - & - & - & 0.25 \\
\hline $\mathrm{Fe} 1$ & $0.9995(12)$ & 0 & $0.2549(6)$ & $0.03(1)$ & 0 & 0 & $0.2546(6)$ & $0.05(1)$ & 0 & 0 & $0.2543(6)$ & $0.10(1)$ \\
\hline $\mathrm{Fe} 2(1)$ & $\frac{1}{4}$ & $\frac{1}{4}$ & $\frac{1}{4}$ & 0.03 & $\frac{1}{4}$ & $\frac{1}{4}$ & $\frac{1}{4}$ & 0.05 & $\frac{1}{4}$ & $\frac{1}{4}$ & $\frac{1}{4}$ & 0.10 \\
\hline $\mathrm{Fe} 2(2)$ & $\frac{1}{4}$ & $\frac{1}{4}$ & $\frac{3}{4}$ & 0.03 & - & - & - & - & - & - & - & - \\
\hline $\mathrm{Fe} 3$ & $0.5005(13)$ & 0 & 0.250 & 0.03 & $\frac{1}{2}$ & 0 & $\frac{1}{4}$ & 0.05 & $\frac{1}{2}$ & 0 & $\frac{1}{4}$ & 0.10 \\
\hline $\mathrm{O} 1$ & 0 & 0 & $\frac{1}{2}$ & $0.25(2)$ & 0 & 0 & $\frac{1}{2}$ & $0.30(1)$ & 0 & 0 & $\frac{1}{2}$ & $0.42(1)$ \\
\hline $\mathrm{O} 2(1)$ & $0.1212(10)$ & $0.1176(12)$ & $0.2127(8)$ & 0.25 & $0.1201(3)$ & 0.1201 & $0.2227(4)$ & 0.30 & $0.1203(3)$ & 0.1203 & $0.2228(4)$ & 0.42 \\
\hline $\mathrm{O} 2(2)$ & $0.1173(11)$ & $0.1221(11)$ & $0.7618(10)$ & 0.25 & - & - & - & 0.30 & - & - & - & 0.42 \\
\hline $\mathrm{O} 3$ & $0.2393(4)$ & 0.2393 & $0.5027(21)$ & 0.25 & $0.2398(4)$ & 0.2398 & $\frac{1}{2}$ & 0.30 & $0.2398(4)$ & 0.2398 & $\frac{1}{2}$ & 0.42 \\
\hline $\mathrm{O} 4(1)$ & $0.1261(12)$ & $0.6210(17)$ & $0.2496(19)$ & 0.25 & $0.1238(5)$ & 0.6238 & $\frac{1}{4}$ & 0.30 & $0.1237(4)$ & 0.6237 & $\frac{1}{4}$ & 0.42 \\
\hline $\mathrm{O} 4(2)$ & $0.1293(12)$ & $0.6204(17)$ & $0.7439(16)$ & 0.25 & - & - & - & 0.30 & - & - & - & 0.42 \\
\hline O5(1) & $\frac{1}{2}$ & 0 & 0 & 0.25 & $\frac{1}{2}$ & 0 & 0 & 0.30 & $\frac{1}{2}$ & 0 & 0 & 0.42 \\
\hline $\mathrm{O} 5(2)$ & 0 & $\frac{1}{2}$ & 0 & 0.25 & - & - & - & 0.30 & - & - & - & 0.42 \\
\hline
\end{tabular}

$4 d\left(\frac{1}{2}, 0, \frac{1}{4}\right), \mathrm{O} 1$ in $2 b\left(0,0, \frac{1}{2}\right), \mathrm{O} 2$ in $16 m(x, x, z), \mathrm{O} 3$ in $8 h\left(x, x, \frac{1}{2}\right)$, $\mathrm{O} 4$ in $16 k\left(x, x+\frac{1}{2}, \frac{1}{4}\right)$, and $\mathrm{O} 5$ in $4 c\left(\frac{1}{2}, 0,0\right)$.

In Fig. 1 the crystal structure of $\mathrm{Sr}_{8} \mathrm{Fe}_{8} \mathrm{O}_{23}$ is compared with the structures of the cubic and orthorhombic phases $\mathrm{SrFeO}_{3}$ and $\mathrm{Sr}_{4} \mathrm{Fe}_{4} \mathrm{O}_{11}$, respectively. $\mathrm{Sr}_{4} \mathrm{Fe}_{4} \mathrm{O}_{11}$ (space group Cmmm) is characterized by corner-sharing square pyramids $\left(\mathrm{FeO}_{5}\right)$ along the orthorhombic $c$ axis. Due to the oxygen deficiency at one of the apical oxygen sites, the Fe atom at $4 i(0, y, 0)$ is shifted out of the plane [or from the ideal value $y=\frac{1}{4}$ to $0.253(1)$ ], which is formed by the four equatorial oxygen atoms. This can be attributed to the strong bond between the iron atom and the apical atom $\mathrm{O} 1$, which is fixed at the position $2 b\left(0, \frac{1}{2}, 0\right)$ in $C m m m .^{6,8}$ Note that the $b$ axis of the orthorhombic setting corresponds to the $c$ axis in the tetragonal one (Fig. 1). The same behavior was observed for the $\mathrm{FeO}_{5}$ units in $\mathrm{Sr}_{8} \mathrm{Fe}_{8} \mathrm{O}_{23}$, where the $z$ parameter of the $\mathrm{Fe} 1$ atom [at $4 e(0,0, z)]$ in the $\mathrm{FeO}_{5}$ unit is shifted from the ideal value $z=$ $\frac{1}{4}$ to $0.2546(6)$ at $90 \mathrm{~K}$ and $0.2543(6)$ at $199 \mathrm{~K}$, respectively. This Fe1 atom is then also closer to the apical $\mathrm{O} 1$ atom, which is fixed at the position $2 b\left(0,0, \frac{1}{2}\right)$ in $I 4 / \mathrm{mmm}$ (Table I). Our results disagree with a previous report on the roomtemperature structure of $\mathrm{Sr}_{8} \mathrm{Fe}_{8} \mathrm{O}_{23}$, which assigned the ideal position of 0.250 (2) to the Fel atom. ${ }^{6}$ This result is surprising, because our refinements showed that the $z$ parameter of Fe1 does not change significantly between 90 and $199 \mathrm{~K}$ (Table I). The equatorial oxygen atoms in both $\mathrm{Sr}_{4} \mathrm{Fe}_{4} \mathrm{O}_{11}$ and $\mathrm{Sr}_{8} \mathrm{Fe}_{8} \mathrm{O}_{23}$ show shifts in the direction opposite to one of the iron atoms: $\mathrm{O} 3[16 r(x, y, z)]$ in $\mathrm{Sr}_{4} \mathrm{Fe}_{4} \mathrm{O}_{11}$ from $y=$ $\frac{1}{4}$ to $0.2239(1) ; \mathrm{O} 2[16 m(x, x, z)]$ in $\mathrm{Sr}_{8} \mathrm{Fe}_{8} \mathrm{O}_{23}$ from $z=$ $\frac{1}{4}$ to $0.2227(4)$ at $90 \mathrm{~K}$ and $0.2218(4)$ at $199 \mathrm{~K}$. These distortions of the square-pyramidal $\mathrm{FeO}_{5}$ units induce tilts of the corner-shared $\mathrm{FeO}_{6}$ octahedra, as shown in Fig. 1.

For a detailed investigation of the structural modifications associated with the charge-ordering transition at $T_{\mathrm{S}}=75 \mathrm{~K}$, we used powder patterns collected at 2 and $90 \mathrm{~K}$ (Fig. 7). The crystal structure of $\mathrm{Sr}_{8} \mathrm{Fe}_{8} \mathrm{O}_{23}$ at $90 \mathrm{~K}$ could be again refined in the space group $I 4 / \mathrm{mmm}$, resulting in a residual $R_{F}=0.034$ $\left(\chi^{2}=1.45\right)$, which is similar to that obtained from the data set collected at $199 \mathrm{~K}$. The refinement of the structure at $2 \mathrm{~K}$ in the tetragonal space group resulted in a similar residual $R_{F}=$ 0.037 , but in a considerably larger $\chi^{2}$ value $\left(\chi^{2}=2.24\right)$. In contrast to the powder pattern collected at $90 \mathrm{~K}$, it could be seen that some of the refined peak positions in the $2 \theta$ range above $130^{\circ}$ differed from the observed positions. As shown in the inset of Fig. 7, the $2 \theta$ positions of reflections $(h, k, \ell)$ with large $\ell$ showed the strongest discrepancies. This led us to refine the crystal structure of $\mathrm{Sr}_{8} \mathrm{Fe}_{8} \mathrm{O}_{23}$ in a lower-symmetric space group. Among all the space groups that could be deduced from group-subgroup relations, the best fit was obtained for the monoclinic space group $I 2 / m$ (No. 12 , setting $I 12 / m 1$, standard setting $C 2 / \mathrm{m})$ resulting in a residual $R_{F}=0.037\left(\chi^{2}=\right.$ 1.92). The observed and calculated powder patterns at 2 and $90 \mathrm{~K}$ are shown in Fig. 7.

Due to the symmetry reduction, the Wyckoff positions of the atoms $\mathrm{Sr} 1, \mathrm{Sr} 2, \mathrm{Fe} 2, \mathrm{O} 2, \mathrm{O} 3, \mathrm{O} 4$, and $\mathrm{O} 5$ in $14 / \mathrm{mmm}$ split into two different sites: $\operatorname{Sr} 11$ in $4 i(x, 0, z)$ and $\operatorname{Sr} 12$ in $4 g(0, y, 0)$, $\mathrm{Sr} 21$ in $4 i(x, 0, z)$ and $\mathrm{Sr} 22$ in $4 h\left(0, y, \frac{1}{2}\right), \mathrm{Fe} 21$ in $4 e\left(\frac{1}{4}, \frac{1}{4}, \frac{1}{4}\right)$ and $\mathrm{Fe} 22$ in $4 f\left(\frac{1}{4}, \frac{1}{4}, \frac{3}{4}\right), \mathrm{O} 21$ and $\mathrm{O} 22$ as well as $\mathrm{O} 41$ and $\mathrm{O} 42$ in $8 j(x, y, z), \mathrm{O} 51$ in $2 b\left(0, \frac{1}{2}, 0\right)$ and 052 in $2 c\left(\frac{1}{2}, 0,0\right)$. For the atoms $\mathrm{Fe} 1, \mathrm{Fe} 3$, and $\mathrm{O} 3$ the multiplicities are retained: $\mathrm{Fe} 1$ and $\mathrm{Fe} 3$ in $4 i(x, 0, z), \mathrm{O} 3$ in $8 j(x, y, z)$. In the tetragonal structure $(I 4 / \mathrm{mmm})$ a total number of seven positional parameters were refined, while in the lower-symmetric space group $I 2 / \mathrm{m}$ a much larger number of 25 positional parameters were allowed to vary. This was problematic within $I 2 / \mathrm{m}$, as the parameters of the monoclinic phase are highly correlated. As a consequence, the parameters of the monoclinic structure could not be determined as accurately as those of the tetragonal 

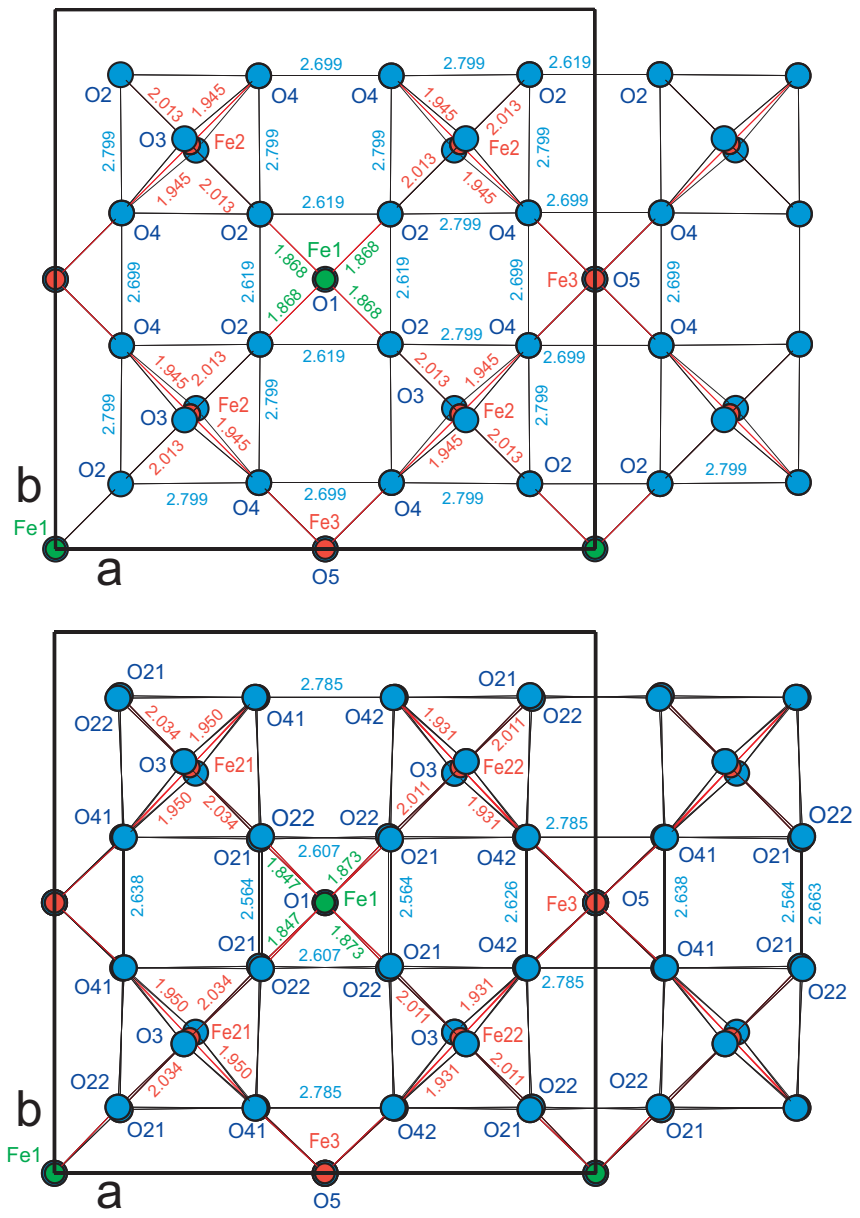

FIG. 8. (Color online) Projection of the high-temperature tetragonal (top) and the low-temperature monoclinic (bottom) crystal structure of $\mathrm{Sr}_{8} \mathrm{Fe}_{8} \mathrm{O}_{23}$ along [001]. The network of distorted $\mathrm{FeO}_{6}$ octahedra in the $a b$ plane is shown at the level $z=0.25$. In order to see the displacements of the $\mathrm{O} 2$ atoms in the monoclinic structure, the $\mathrm{O} 2$ atoms of the neighboring $\mathrm{FeO}_{6}$ octahedra at $z=0.75$ are also shown. The values of particular interatomic $\mathrm{Fe}-\mathrm{O}$ and $\mathrm{O}-\mathrm{O}$ bond distances are also given.

phase (Table I). However, the refinements showed that the $z$ parameter of $\mathrm{Fe} 3$ is practically the same as the ideal value $\frac{1}{4}$ that is fixed in $I 4 / \mathrm{mmm}$. Further the $x$ and $y$ parameters of $\mathrm{O} 3$ were found to be identical. Therefore in the last refinement the value $z(\mathrm{Fe} 3)$ was fixed at $\frac{1}{4}$, and $x(\mathrm{O} 3)$ and $y(\mathrm{O} 3)$ were constrained to be equal.

The positional parameters and the distances between the oxygen and iron atoms obtained at 2, 90, and $199 \mathrm{~K}$ are listed in Tables I and II, and the $a b$-plane projections of the hightemperature tetragonal and the low-temperature monoclinic structures of $\mathrm{Sr}_{8} \mathrm{Fe}_{8} \mathrm{O}_{23}$ are shown in Fig. 8. The octahedrally coordinated $\mathrm{Fe} 2$ atoms are tilted in the tetragonal phase due the oxygen vacancies at the $\mathrm{Fe} 1$ sites. Due to the tetragonal symmetry, the apical $\mathrm{O} 3$ atoms [in $\left.8 h\left(x, x, \frac{1}{2}\right)\right]$ are shifted along the directions [110] and [1-10] or inverse. In the monoclinic structure, the independent parameters $x$ and $y$ of the $\mathrm{O} 3$ atoms [in $8 j(x, y, z)$ ] did not show any significant shift from the positions obtained for the tetragonal phase. In Fig. 8 it can be seen that the tilts of the $\mathrm{FeO}_{6}$ octahedra are not significantly

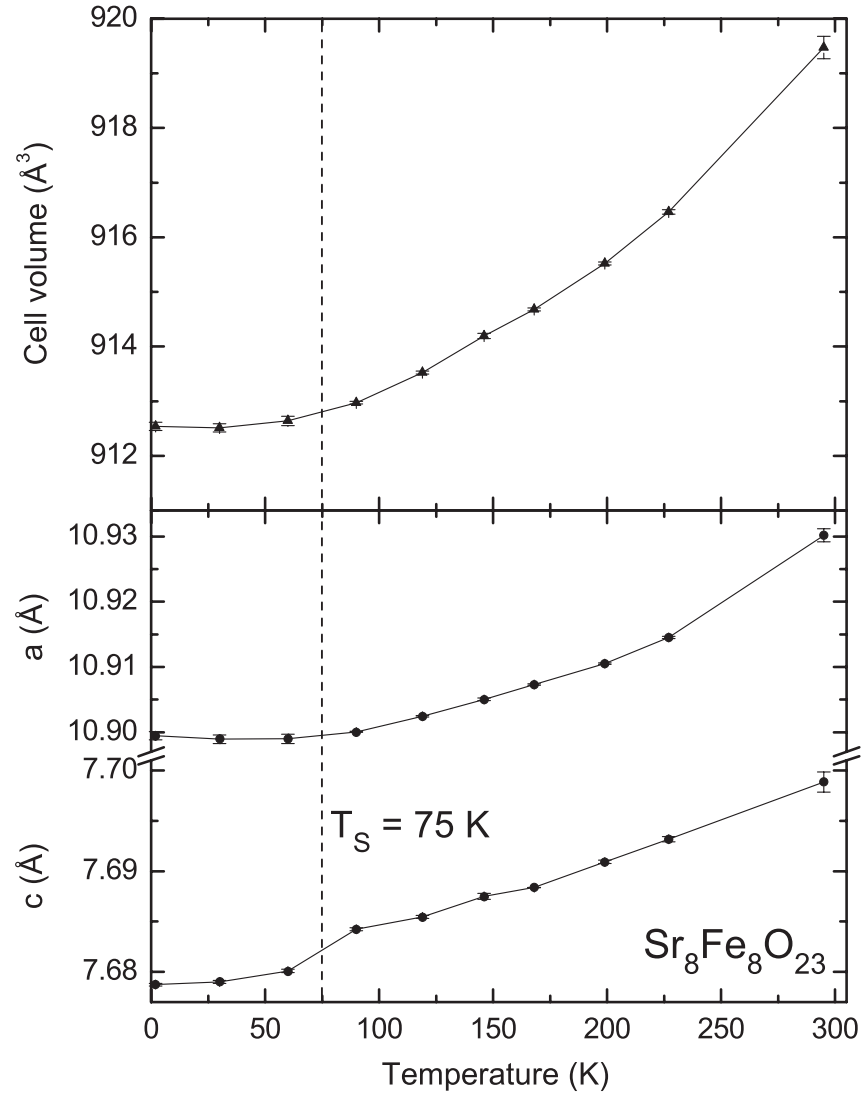

FIG. 9. Temperature dependence of the lattice parameters of $\mathrm{Sr}_{8} \mathrm{Fe}_{8} \mathrm{O}_{23}$. With decreasing temperature the tetragonal structure of $\mathrm{Sr}_{8} \mathrm{Fe}_{8} \mathrm{O}_{23}$ changes to a monoclinic structure at the transition temperature $T_{\mathrm{S}}=75(2) \mathrm{K}$. A significant decrease could be observed for the $c$ parameter, while the $a$ parameter shows only a slight increase below $30 \mathrm{~K}$. The $b$ parameter (monoclinic axis) does not show any significant change in the monoclinic phase between 2 and $60 \mathrm{~K}$.

influenced by the change of symmetry. The main changes in the monoclinic structure occur in the $a b$ plane. The $\mathrm{O} 2$ atoms in the tetragonal structure are located at the Wyckoff position $\mathrm{O} 2$ in $16 m(x, x, z)$, while in the monoclinic setting this position is split into two positions $\mathrm{O} 21$ and $\mathrm{O} 22$, both in $8 j(x, y, z)$. In Table I it can be seen that the refined parameters $x(\mathrm{O} 21)$ and $y(\mathrm{O} 22)$ as well as $y(\mathrm{O} 21)$ and $x(\mathrm{O} 22)$ are similar. Figure 8 shows that the $\mathrm{O} 21$ and $\mathrm{O} 22$ atoms are shifted parallel to the directions [110] and [1-10] (or inverse) which are almost perpendicular to the $\mathrm{Fe}-\mathrm{O}$ bonds. On the other hand, the $x$ and $y$ parameters of the other equatorial $\mathrm{O} 41$ and $\mathrm{O} 42$ atoms were found to be similar.

Additional data sets were collected in the monoclinic phase at 30 and $60 \mathrm{~K}$, as well as in the tetragonal phase at 119,146 , 168 , and $227 \mathrm{~K}$. The refinements showed that the structural parameters of the monoclinic phase up to $60 \mathrm{~K}$ are similar to those obtained from the powder pattern collected at $2 \mathrm{~K}$. This shows that the monoclinic structure is already well established $15 \mathrm{~K}$ below $T_{\mathrm{S}}=75 \mathrm{~K}$. From all these data sets we were able to determine the lattice parameters with good accuracy. In Fig. 9 it can be seen that the lattice parameters $a(=b)$ and $c$ show a continuous decrease in the tetragonal phase down to $90 \mathrm{~K}$. Below the structural phase transition, only the $c$ parameter shows a significant change. 
We now discuss the implications of these observations for our understanding of the charge-ordering transition in $\mathrm{Sr}_{8} \mathrm{Fe}_{8} \mathrm{O}_{23}$. At room temperature, this compound contains three different iron sites, namely $\mathrm{Fe} 1$ with square-pyramidal coordination and two crystallographically distinct atoms $\mathrm{Fe} 2$ and Fe3 with octahedral coordination, with a ratio of 1:2:1. Mössbauer spectra at room temperature showed two different components, $\mathrm{Fe}^{3.5+}$ and $\mathrm{Fe}^{4+}$, with a ratio of $1: 1$ (Refs. 11 and 12), and calculations of the $\mathrm{Fe}-\mathrm{O}$ bond-strength sums around the $\mathrm{Fe}$ sites indicated that the $\mathrm{Fe} 2$ ions possess a lower valence state than the $\mathrm{Fe} 1$ and $\mathrm{Fe} 3$ ions. ${ }^{6}$ Based on this information, the $\mathrm{Fe}^{4+}$ state could be assigned to both the $\mathrm{Fe} 1$ and the $\mathrm{Fe} 3$ atoms, while the $\mathrm{Fe} 2$ atoms are in the valence state $\mathrm{Fe}^{3.5+}$.

We first focus on the behavior of the $\mathrm{Fe}^{4+}$ ions. The almost regular $\mathrm{Fe}_{3} \mathrm{O}_{6}$ octahedron in $\mathrm{Sr}_{8} \mathrm{Fe}_{8} \mathrm{O}_{23}$ is reminiscent of the undistorted octahedra in cubic $\mathrm{SrFeO}_{3}$, where the $\mathrm{Fe}-\mathrm{O}$ bond distance is $d(\mathrm{Fe}-\mathrm{O})=1.9255(5) \AA$ (at room temperature). In orthorhombic $\mathrm{CaFeO}_{3}$ the three different $d(\mathrm{Fe}-\mathrm{O})$ values vary between 1.9184(6) and 1.927(2) $\AA$ [averaged $d_{\mathrm{av}}(\mathrm{Fe}-\mathrm{O})=$ 1.922(2) $\AA$ ].$^{6,25}$ In tetragonal $\mathrm{Sr}_{8} \mathrm{Fe}_{8} \mathrm{O}_{23}$ the averaged bond distance $d_{\mathrm{av}}(\mathrm{Fe} 3-\mathrm{O})=1.916(2) \AA$ (at room temperature) was found to be slightly smaller. ${ }^{6}$ We found very similar values for the tetragonal phase $\mathrm{Sr}_{8} \mathrm{Fe}_{8} \mathrm{O}_{23}$ at lower temperatures: $d_{\mathrm{av}}(\mathrm{Fe} 3-\mathrm{O})=1.914(4) \AA$ at $199 \mathrm{~K}$ and $1.913(4) \AA$ at $90 \mathrm{~K}$. In the monoclinic phase, neither the average $\mathrm{Fe} 3-\mathrm{O}$ distance $\left[d_{\mathrm{av}}(\mathrm{Fe} 3-\mathrm{O})=1.917(7) \AA\right.$ at $\left.2 \mathrm{~K}\right]$ nor the shape of the $\mathrm{Fe} 3 \mathrm{O}_{6}$ units differ significantly from the tetragonal phase. In agreement with prior Mössbauer spectroscopy results (Refs. 11 and 12), we therefore conclude that the Fe3 atoms do not participate in the charge-ordering transition. The same conclusions hold for the $\mathrm{Fe} 1$ atoms, which are also in the $\mathrm{Fe}^{4+}$ valence state. The average $\mathrm{Fe} 1-\mathrm{O}$ bond length in $\mathrm{Sr}_{8} \mathrm{Fe}_{8} \mathrm{O}_{23}$, $d_{\mathrm{av}}(\mathrm{Fe} 1-\mathrm{O})=1.864(8) \AA$ at $2 \mathrm{~K}$, is the same as that in the square-pyramidal $\mathrm{FeO}_{5}$ units in $\mathrm{Sr}_{4} \mathrm{Fe}_{4} \mathrm{O}_{11} \cdot 6,8$ Again, only minor changes were observed at the charge-ordering transition.

In contrast to the $\mathrm{Fe} 1$ and $\mathrm{Fe} 3$ atoms of $\mathrm{Sr}_{8} \mathrm{Fe}_{8} \mathrm{O}_{23}$, the Fe2-O bonds are modified substantially at the charge-ordering transition. In the tetragonal charge-disordered phase, two of the six $\mathrm{Fe} 2-\mathrm{O}$ bonds in the $\mathrm{Fe} 2 \mathrm{O}_{6}$ octahedron are significantly elongated (Table II and Fig. 8), probably at least in part because of steric constraints imposed by adjacent oxygen vacancies. In the monoclinic charge-ordered phase, the Fe2 site splits into two different sites, Fe21 and Fe22, with different averaged bond lengths $d_{\mathrm{av}}(\mathrm{Fe} 21-\mathrm{O})=1.977(11) \AA$ and $d_{\mathrm{av}}(\mathrm{Fe} 22-\mathrm{O})=$ $1.949(11) \AA$, which are larger and smaller than the value $d_{\mathrm{av}}(\mathrm{Fe} 22-\mathrm{O})=1.962(3) \AA$ obtained at $90 \mathrm{~K}$, respectively. This is qualitatively consistent with a charge-ordering transition in the two-dimensional $\mathrm{Fe} 2$ atom network, in agreement with the conclusions from Mössbauer spectroscopy. ${ }^{11,12}$ However, the difference in the averaged bond lengths of Fe21 and Fe22 atoms is only about half of the value $\Delta d=0.055 \AA$, the difference of the bond distances of octahedrally coordinated $\mathrm{Fe}$ atoms in the valence states $\mathrm{Fe}^{3+}$ and $\mathrm{Fe}^{4+}$ given by Shannon. ${ }^{26}$ Note also that the difference in averaged bond lengths of the $\mathrm{Fe}$ atoms with formal valence $\mathrm{Fe}^{3+}$ and $\mathrm{Fe}^{5+}$ in the monoclinic, charge-disproportionated phase of $\mathrm{CaFeO}_{3}$ is even larger: $\Delta d=0.102 \AA .^{25}$ This comparison indicates incomplete charge segregation in the monoclinic phase of $\mathrm{Sr}_{8} \mathrm{Fe}_{8} \mathrm{O}_{23}$. Similar conclusions have been reported for other charge-ordered transition metal oxides, including in particular the charge-disproportionated state of $\mathrm{La}_{1 / 3} \mathrm{Sr}_{2 / 3} \mathrm{FeO}_{3} .{ }^{27} \mathrm{We}$ also note that the $\mathrm{Fe} 2-\mathrm{O}$ bond-length modification in the charge-ordered state is anisotropic. In particular, only the two Fe21-O21 bonds increase significantly below the transition, while the other four bond lengths $[d(\mathrm{Fe} 21-\mathrm{O} 41)$ and $d(\mathrm{Fe} 21-$ $\mathrm{O} 3)]$ in the $(\mathrm{Fe} 21) \mathrm{O}_{6}$ octahedon are almost identical (Table II). In contrast, the elongated Fe22-O22-bonds do not show any significant change between the tetragonal and monoclinic structures. This indicates that charge ordering is associated with some degree of orbital polarization, which differs between the nominal $\mathrm{Fe}^{3+}$ and $\mathrm{Fe}^{4+}$ valence states, as expected. Detailed electronic-structure calculations will be required to explain the specific bond-distortion pattern we have observed.

\section{Magnetic order in $\mathrm{SrFeO}_{3-\delta}$ \\ 1. Phase I}

We now discuss some of the magnetic states identified in Sec. III A in more detail. We begin with the stoichiometric end member $\mathrm{SrFeO}_{3}$, where helical magnetic order with a propagation vector $\boldsymbol{k}=(\Delta, \Delta, \Delta)_{\text {cub }}$ and incommensurability $\Delta=0.112$ was found earlier by Takeda et al. ${ }^{1}$ In this study the magnetic moment of the iron atoms, $\mu_{\exp }=2.7(4)$ $\mu_{\mathrm{B}}$, was determined at the relatively high temperature of $77 \mathrm{~K}$. In order to accurately determine the saturation value of the magnetic moment, we have reinvestigated the magnetic structure $\mathrm{SrFeO}_{3}$ at $T=2 \mathrm{~K}$ by single-crystal neutron diffraction using the neutron diffractometer D10 at the ILL in Grenoble and the triple-axis spectrometer Rita-II at the PSI in Villigen/Switzerland. For the experiment we used a single crystal with the composition $\mathrm{SrFeO}_{3.00}$ with dimensions $3 \times 6 \times 8 \mathrm{~mm}^{3}$. At the diffractometer D10 we first collected a set of 122 (nine unique) nuclear Bragg reflections in order to determine the overall scale factor from the crystal structure refinement, which is needed for the determination of the precise value of the ordered iron moment. In the cubic perovskite structure of $\mathrm{SrFeO}_{3}$ with the space group $P m \overline{3} m$ the $\mathrm{Sr}, \mathrm{Fe}$, and $\mathrm{O}$ atoms are located at the following Wyckoff positions: $\mathrm{Sr}$ in $1 b\left(\frac{1}{2}, \frac{1}{2}, \frac{1}{2}\right), \mathrm{Fe}$ in $1 a(0,0,0), \mathrm{O}$ in $3 d\left(0,0, \frac{1}{2}\right)$, where no positional parameters are refinable. Due to the fact that we only collected reflections up to $\sin \theta / \lambda=0.39 \AA^{-1}$ the temperature factors could not be determined with good accuracy. Therefore we used for the refinements a fixed value $B=0.3 \AA^{2}$ for all the atoms $\mathrm{Sr}, \mathrm{Fe}$, and $\mathrm{O}$. The refinements of the overall scale factor and the extinction parameters resulted in a satisfactory residual of $R_{F}=0.020$.

For the refinement of the magnetic structure of $\mathrm{SrFeO}_{3}$ a set of 143 (28 unique) satellites was collected. The analysis confirmed the presence of a helical magnetic ordering of the $\mathrm{Fe}^{4+}$ sublattice as found earlier. ${ }^{1}$ Using the large number of absorption- and extinction-corrected magnetic structure factors, we were able to determine the magnetic moment with very good accuracy, as documented by the residual $R_{F}=$ 0.040. The refined magnetic moment of the iron atoms was found to be $\mu_{\exp }=2.960(12) \mu_{\mathrm{B}}$ at $2 \mathrm{~K}$. This value is larger than the value $\mu_{\exp }=2.7(4) \mu_{\mathrm{B}}$ obtained earlier at $77 \mathrm{~K}$ (Ref. 1), and it could be determined with improved precision. The total moment of the $\mathrm{Fe}^{4+}$ ions $\left(3 d^{4}\right.$-electron configuration) is well below the free-ion value of $4.0 \mu_{\mathrm{B}}$. While this is not unexpected for a metallic compound, we note that the ordered 
TABLE II. Interatomic distances (in $\AA$ ) between the oxygen and iron atoms in tetragonal and monoclinic $\mathrm{Sr}_{8} \mathrm{Fe}_{8} \mathrm{O}_{23}$. The $\mathrm{Fe} 2$ and $\mathrm{Fe} 3$ atoms show an octahedral coordination $\left(\mathrm{FeO}_{6}\right)$. Due the oxygen deficiency the $\mathrm{Fe} 1$ atom shows a square-pyramidal coordination $\left.(\mathrm{FeO})_{5}\right)$. The lattice constants, cell volumes, and bond angles of $\mathrm{Sr}_{8} \mathrm{Fe}_{8} \mathrm{O}_{23}$ are also given. The values determined at 2, 90, and $199 \mathrm{~K}$ are compared with the room temperature values presented by Hodges et al. (Ref. 6).

\begin{tabular}{|c|c|c|c|c|}
\hline $\mathrm{Sr}_{8} \mathrm{Fe}_{8} \mathrm{O}_{23}$ & at $2 \mathrm{~K}(I 2 / m)$ & at $90 \mathrm{~K}(I 4 / \mathrm{mmm})$ & at $199 \mathrm{~K}(I 4 / \mathrm{mmm})$ & at RT $(I 4 / \mathrm{mmm})$ \\
\hline$d_{\mathrm{Fe} 1-\mathrm{O} 21}$ & $1.873(9) \times 2$ & $1.868(3) \times 4$ & $1.871(3) \times 4$ & $1.851(4) \times 4$ \\
\hline$d_{\mathrm{Fe} 1-\mathrm{O} 22}$ & $1.847(9) \times 2$ & 1.868 & 1.871 & 1.851 \\
\hline$d_{\mathrm{Fe} 1-\mathrm{O} 1}$ & $1.882(5) \times 1$ & $1.886(4) \times 1$ & $1.889(3) \times 1$ & $1.926(1) \times 1$ \\
\hline$d_{\mathrm{O} 1-\mathrm{O} 21}$ & $2.874(8) \times 2$ & $2.823(3) \times 4$ & $2.826(3) \times 4$ & $2.820(3) \times 4$ \\
\hline$d_{\mathrm{O} 1-\mathrm{O} 22}$ & $2.729(8) \times 2$ & 2.823 & 2.826 & 2.820 \\
\hline$d_{\mathrm{O} 21-\mathrm{O} 21}$ & $2.564(18) \times 1$ & $2.619(4) \times 4$ & $2.624(4) \times 4$ & $2.601(4) \times 4$ \\
\hline$d_{\mathrm{O} 22-\mathrm{O} 22}$ & $2.663(18) \times 1$ & 2.619 & 2.624 & 2.601 \\
\hline$d_{\mathrm{O} 21-\mathrm{O} 22}$ & $2.608(16 \times 2$ & 2.619 & 2.624 & 2.601 \\
\hline$d_{\mathrm{Fe} 21-\mathrm{O} 21}$ & $2.034(9) \times 2$ & $2.013(3) \times 2$ & $2.013(3) \times 2$ & $2.036(3) \times 2$ \\
\hline$d_{\mathrm{Fe} 21-\mathrm{O} 41}$ & $1.950(9) \times 2$ & $1.945(5) \times 2$ & $1.948(6) \times 2$ & $1.952(3) \times 2$ \\
\hline$d_{\mathrm{Fe} 21-\mathrm{O} 3}$ & $1.947(16) \times 2$ & $1.9274(3) \times 2$ & $1.9292(4) \times 2$ & $1.931(1) \times 2$ \\
\hline$d_{\mathrm{O} 21-\mathrm{O} 3}$ & $2.737(13) \times 2$ & $2.755(4) \times 2$ & $2.757(5) \times 2$ & $2.772(5) \times 2$ \\
\hline$d_{\mathrm{O} 21-\mathrm{O} 3}$ & $2.893(14) \times 2$ & $2.819(4) \times 2$ & $2.819(4) \times 2$ & $2.839(4) \times 2$ \\
\hline$d_{\mathrm{O} 41-\mathrm{O} 3}$ & $2.753(15) \times 2$ & $2.738(5) \times 2$ & $2.742(5) \times 2$ & $2.745(2) \times 4$ \\
\hline$d_{\mathrm{O} 41-\mathrm{O} 3}$ & $2.759(15) \times 2$ & $2.738(5) \times 2$ & $2.742 \times 2$ & 2.745 \\
\hline$d_{\mathrm{O} 21-\mathrm{O} 41}$ & $2.770(16) \times 2$ & $2.799(6) \times 2$ & $2.801(6) \times 2$ & $2.820(3) \times 4$ \\
\hline$d_{\mathrm{O} 21-\mathrm{O} 41}$ & $2.865(9) \times 2$ & $2.799 \times 2$ & $2.801 \times 2$ & 2.820 \\
\hline$d_{\mathrm{Fe} 22-\mathrm{O} 22}$ & $2.011(9) \times 2$ & $2.013(3) \times 2$ & $2.013(3) \times 2$ & $2.036(3) \times 2$ \\
\hline$d_{\mathrm{Fe} 22-\mathrm{O} 42}$ & $1.931(9) \times 2$ & $1.945(5) \times 2$ & $1.948(6) \times 2$ & $1.952(3) \times 2$ \\
\hline$d_{\mathrm{Fe} 22-\mathrm{O} 3}$ & $1.906(16) \times 2$ & $1.9274(3) \times 2$ & $1.9292(4) \times 2$ & $1.931(1) \times 2$ \\
\hline$d_{\mathrm{O} 22-\mathrm{O} 3}$ & $2.714(14) \times 2$ & $2.755(4) \times 2$ & $2.757(5) \times 2$ & $2.772(5) \times 2$ \\
\hline$d_{\mathrm{O} 22-\mathrm{O} 3}$ & $2.826(13) \times 2$ & $2.819(4) \times 2$ & $2.819(4) \times 2$ & $2.839(4) \times 2$ \\
\hline$d_{\mathrm{O} 42-\mathrm{O} 3}$ & $2.686(15) \times 2$ & $2.738(5) \times 2$ & $2.742(5) \times 2$ & $2.745(2) \times 4$ \\
\hline$d_{\mathrm{O} 42-\mathrm{O} 3}$ & $2.741(16) \times 2$ & $2.838(4) \times 2$ & $2.742 \times 2$ & 2.745 \\
\hline$d_{\mathrm{O} 22-\mathrm{O} 42}$ & $2.762(14) \times 2$ & $2.799(6) \times 2$ & $2.801(6) \times 2$ & $2.820(3) \times 4$ \\
\hline$d_{\mathrm{O} 22-\mathrm{O} 42}$ & $2.813(13) \times 2$ & $2.799 \times 2$ & $2.801 \times 2$ & 2.820 \\
\hline$d_{\mathrm{Fe} 3-\mathrm{O} 41}$ & $1.909(9) \times 2$ & $1.909(5) \times 4$ & $1.909(5) \times 4$ & $1.912(3) \times 4$ \\
\hline$d_{\mathrm{Fe} 3-\mathrm{O} 42}$ & $1.923(10) \times 2$ & 1.909 & 1.909 & 1.912 \\
\hline$d_{\mathrm{Fe} 3-\mathrm{O} 5}$ & $1.920(1) \times 2$ & $1.9211(3) \times 2$ & $1.9227(3) \times 2$ & $1.925(1) \times 2$ \\
\hline$d_{\mathrm{O} 41-\mathrm{O} 51}$ & $2.708(10) \times 2$ & $2.708(4) \times 8$ & $2.710(4) \times 8$ & $2.713(2) \times 8$ \\
\hline$d_{\mathrm{O} 41-\mathrm{O} 52}$ & $2.701(10) \times 2$ & 2.708 & 2.710 & 2.713 \\
\hline$d_{\mathrm{O} 42-\mathrm{O} 11}$ & $2.685(10) \times 2$ & 2.708 & 2.710 & 2.713 \\
\hline$d_{\mathrm{O} 42-\mathrm{O} 52}$ & $2.755(10) \times 2$ & 2.708 & 2.710 & 2.713 \\
\hline$d_{\mathrm{O} 41-\mathrm{O} 41}$ & $2.638(8) \times 1$ & $2.699(8) \times 4$ & $2.700(8) \times 4$ & $2.704(4) \times 4$ \\
\hline$d_{\mathrm{O} 42-\mathrm{O} 42}$ & $2.626(8) \times 1$ & 2.699 & 2.700 & 2.704 \\
\hline$d_{\mathrm{O} 41-\mathrm{O} 42}$ & $2.785(17) \times 2$ & 2.699 & 2.700 & 2.704 \\
\hline$d_{\mathrm{av}(\mathrm{Fe} 1-\mathrm{O})}$ & $1.864(8)$ & $1.871(3)$ & $1.875(4)$ & $1.866(2)$ \\
\hline$d_{\mathrm{av}(\mathrm{Fe} 21-\mathrm{O})}$ & $1.977(11)$ & $1.962(3)$ & $1.963(3)$ & $1.976(2)$ \\
\hline$d_{\mathrm{av}(\mathrm{Fe} 22-\mathrm{O})}$ & $1.949(11)$ & 1.962 & 1.963 & 1.976 \\
\hline$d_{\mathrm{av}(\mathrm{Fe} 3-\mathrm{O})}$ & $1.917(7)$ & $1.913(4)$ & $1.914(4)$ & $1.916(2)$ \\
\hline$a(\AA)$ & $10.8995(6)$ & $10.90005(10)$ & $10.91050(12)$ & $10.929(1)$ \\
\hline$b(\AA)$ & $10.9033(6)$ & 10.90005 & 10.91050 & 10.929 \\
\hline$c(\AA)$ & $7.67873(14)$ & $7.68424(14)$ & $7.69092(16)$ & $7.698(1)$ \\
\hline$\beta\left(^{\circ}\right)$ & $90.0764(25)$ & 90 & 90 & 90 \\
\hline$V\left(\AA^{3}\right)$ & $912.54(8)$ & $912.97(2)$ & $915.52(3)$ & $919.47(21)$ \\
\hline
\end{tabular}

moments of the $\mathrm{Fe}^{3+}$ ions ( $3 d^{5}$-electron configuration) in the insulating charge-ordered ferrites $\mathrm{CaFeO}_{3}$ and $\mathrm{Sr}_{4} \mathrm{Fe}_{4} \mathrm{O}_{11}$ reach the values $\mu_{\exp }=3.5(1) \mu_{\mathrm{B}}$ and $\mu_{\exp }=3.55(5) \mu_{\mathrm{B}}$, respectively, ${ }^{8,26}$ which are also well below the free-ion value of $5.0 \mu_{\mathrm{B}}$. Factors possibly contributing to the moment reduction are charge fluctuations in proximity to the metal-insulator transition, and the strong covalency between the $\mathrm{Fe}$ and $\mathrm{O}$ atoms in the $\mathrm{FeO}_{6}$ and $\mathrm{FeO}_{5}$ units.
Measurements as a function of temperature demonstrate that $\Delta$ decreases with increasing temperature (Fig. 3). This may at first sight appear surprising for a metallic system, where the helix propagation vector is expected to be tied to the dimensions of the Fermi surface. A recent study of $\mathrm{NbSe}_{2}$ has, however, revealed that both the Fermi surface nesting vector and the charge density wave propagation vector depend substantially on temperature, and found a good match between 
both quantities. ${ }^{28,29}$ A temperature-dependent investigation of the Fermi surface of $\mathrm{SrFeO}_{3}$ would therefore be an interesting subject of future work.

Figure 3 also shows that the temperature dependence of the incommensurability does not explain the difference between the saturated value $\Delta=0.129$ found in the present work and the value $\Delta=0.112$ at $T=77 \mathrm{~K}$ reported earlier. ${ }^{1}$ The difference probably reflects a different charge carrier concentration in the sample investigated earlier, possibly due to residual oxygen vacancies. We note, however, that $\Delta$ in the cubic volume fraction of our oxygen-deficient samples does not differ substantially from the one in the $\mathrm{SrFeO}_{3.00}$ sample (Fig. 3, right panel). Rather, the presence of magnetic phase-IV reflections suggests that even a small number of residual oxygen defects segregates in our samples, while they may have been randomly incorporated in the earlier samples, possibly due to a different microstructure caused by a different heat treatment.

\section{Phase II}

The right part of Fig. 2 shows the neutron diffraction data of the single-crystalline sample $\mathrm{SrFeO}_{2.87}$. The data were measured at the triple-axis spectrometer Rita II along the $[1,1,1]_{\text {cub }}$ direction around the nuclear Bragg reflection $(0$,

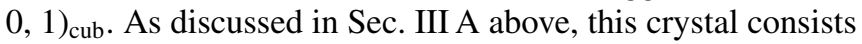
of a phase mixture with predominantly tetragonal $\mathrm{Sr}_{8} \mathrm{Fe}_{8} \mathrm{O}_{23}$ and cubic $\mathrm{SrFeO}_{3}$ with a volume ratio of 74(2):26(2). This is indicated by the appearance of two sets of magnetic Bragg reflections with an incommensurate splitting of $\Delta=0.13$ for the cubic phase, and a second set of Bragg reflections with $\Delta=$ 0.20 that disappear upon heating above the charge-ordering transition, $T_{\mathrm{S}}=75(2) \mathrm{K}$, of the tetragonal phase $\mathrm{Sr}_{8} \mathrm{Fe}_{8} \mathrm{O}_{23}$ (Sec. III B). As found for cubic $\mathrm{SrFeO}_{3}$, the propagation vector of the magnetic "phase II" points along the body diagonal of the cubic unit cell. Only a total number of seven magnetic reflections could be collected on the triple-axis spectrometer. Therefore we were not able to precisely determine the magnetic structure of this phase. However the refinements showed that the moments are aligned perpendicular to the propagation vector $\boldsymbol{k}=(0.20,0.20,0.20)_{\text {cub }}$, indicating a similar helical structure as the one found in the cubic ferrite $\mathrm{SrFeO}_{3}$.

For $T>T_{\mathrm{S}}$, the phase-II satellites evolve into weak diffuse features indicative of correlated phase-II fluctuations in the tetragonal volume fraction of the $\mathrm{SrFeO}_{2.87}$ crystal. Interestingly, the wave vector characterizing these fluctuations deviates from the commensurate value $\Delta=0.20$ and is strongly temperature dependent. With increasing temperature, the diffuse features progressively approach and eventually merge with the Bragg peaks of the helical order in the cubic volume fraction of the crystal. This supports our conclusion that the $\mathrm{SrFeO}_{3}$ and $\mathrm{Sr}_{8} \mathrm{Fe}_{8} \mathrm{O}_{23}$ structures are segregated on a nanoscopic length scale, and that the magnetic ordering phenomena in both phases are influenced by exchange interactions across the phase boundaries.

\section{Phase III}

Our analysis of the data obtained on the powder of composition $\mathrm{SrFeO}_{2.87}$ already discussed in $\mathrm{Sec}$. III B revealed a rather different magnetic ordering pattern in the $\mathrm{Sr}_{8} \mathrm{Fe}_{8} \mathrm{O}_{23}$ volume fraction. Figure 6 shows a series of incommensurate magnetic Bragg reflections at the $2 \theta$ positions $11.2^{\circ}, 13.6^{\circ}$, $18.9^{\circ}$, and $29.6^{\circ}$, which are present only for temperatures below the charge-ordering transition $T_{\mathrm{S}}=75 \mathrm{~K}$ and can therefore be ascribed to the monoclinic structure of $\mathrm{Sr}_{8} \mathrm{Fe}_{8} \mathrm{O}_{23}$. Using the propagation vector $\boldsymbol{k}=(0.687,0,0.326)_{\text {mon }}$ these reflections could be indexed as $(0.687,0,0.326)_{\mathrm{M}},(0.313$, $0,0.674)_{\mathrm{M}},(1.313,0,-0.326)_{\mathrm{M}}$ and $(0.687,2,0.326)_{\mathrm{M}}$. From our high-resolution neutron powder patterns collected at $11 \mathrm{~K}$, we were able to determine the components of the propagation vector with standard deviations of $\sigma= \pm 0.001$. In the cubic notation, the propagation vector can be written as $(0.169,0.169,0.169)_{\text {cub }}$ and is therefore directed along the [111] direction of the cubic unit cell, as found above for phases I and II.

Since magnetic intensity only appears for magnetic reflection with $k=2 n$, antiferromagnetic coupling of the iron atoms with distances $d(\mathrm{Fe}-\mathrm{Fe})=\frac{1}{2} b$ along the monoclinic $b$ axis can be excluded. In order to determine the magnetic structure of this phase of $\mathrm{Sr}_{8} \mathrm{Fe}_{8} \mathrm{O}_{23}$ we first tried the helical model of $\mathrm{SrFeO}_{3}$. In this structure the magnetic moments of the iron atoms form ferromagnetic planes perpendicular to the propagation vector $\boldsymbol{k}$. In the monoclinic setting the real Fourier component is aligned within the $a c$ plane, subtending an angle of $-35.3^{\circ}$ with the $c$ axis. The imaginary Fourier component is set parallel to the monoclinic $b$ axis. With this model, the magnetic intensity of the first reflection $(0.687,0,0.326)_{\mathrm{M}}$ could be well fitted. But in the $2 \theta$ range up to $45^{\circ}$ the calculated magnetic intensities were found to be zero at the positions of the reflections $(0.313,0,0.674)_{\mathrm{M}},(1.313,0,0.326)_{\mathrm{M}}$, and $(0.687,2,0.326)_{\mathrm{M}}$, in disagreement with the experimental data. This suggests that the incommensurate spin structure of the magnetic phase III is different from the helical ordering observed in $\mathrm{SrFeO}_{3}$ and in phase II of $\mathrm{Sr}_{8} \mathrm{Fe}_{8} \mathrm{O}_{23}$. Further trials showed that magnetic intensities could be generated for all four reflections using a model in which the moments of Fe11 in $(0,0,0.255)$ and $\mathrm{Fe} 31$ in $\left(0, \frac{1}{2}, 0.25\right)$ are coupled antiparallel to the moments of the Fe12 atoms in $(0,0,-0.255)$ and Fe32 in $\left(0, \frac{1}{2},-0.25\right)$ (Fig. 10). Note that the atoms $\mathrm{Fe} 11$ and $\mathrm{Fe} 12$ show a square-pyramidal coordination $\left(\mathrm{FeO}_{5}\right)$. This is analogous to the magnetic structure of $\mathrm{Sr}_{4} \mathrm{Fe}_{4} \mathrm{O}_{11}$, where the iron moments are also coupled antiparallel in the dimers of corner-shared $\mathrm{FeO}_{5}$ square pyramids. ${ }^{8}$ However, the refinements did not yield satisfactory agreement between the observed and calculated intensities of all the reflections. Further trials showed that the fit could be considerably improved by refinement of the phase factor $\varphi$ (given in fractions of $2 \pi$ ), which is only allowed to vary for independent sites of magnetically ordered atoms. But a representation analysis following Bertaut (Ref. 30) for the space group I2/m showed that all the atoms Fe1, Fe21, Fe22, and Fe3 split into two orbits. The refined phase factor $\varphi$ between the two sets $(\mathrm{Fe} 11, \mathrm{Fe} 31)$ and $(\mathrm{Fe} 12, \mathrm{Fe} 32)$ resulted in a value close to zero indicating that the iron moments of both sets are aligned almost parallel to each other. For the Fe21 atoms we found the phase factors $\varphi=0.201(11)\left[\mathrm{Fe} 21\right.$ atoms at $4 f:\left(\frac{1}{4}, \frac{1}{4}, \frac{1}{4}\right)$ and $\left.\left(\frac{1}{4},-\frac{1}{4}, \frac{1}{4}\right)\right]$ and $\varphi=0.093(11)\left[\mathrm{Fe} 22\right.$ atoms at $4 e:\left(\frac{1}{4}, \frac{1}{4},-\frac{1}{4}\right)$ and $\left.\left(\frac{1}{4},-\frac{1}{4},-\frac{1}{4}\right)\right]$, which are slightly shifted from the ideal values $\varphi=0.246$ and $\varphi=0.082$ fixed for $\mathrm{SrFeO}_{3}$. In this structure, 


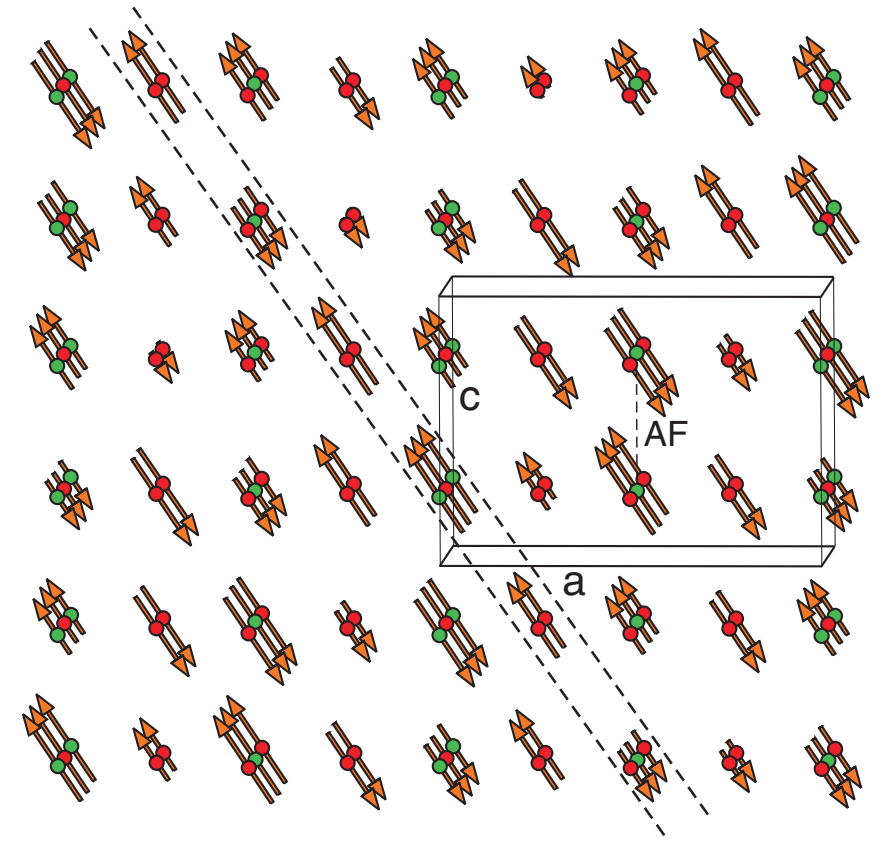

FIG. 10. (Color online) Incommensurate magnetic structure of phase III with the propagation vector $\boldsymbol{k}=(0.687,0,0.326)$, which can be attributed to the magnetic ordering in monoclinic $\mathrm{Sr}_{8} \mathrm{Fe}_{8} \mathrm{O}_{23}$. As found earlier for $\mathrm{SrFeO}_{3}$ the magnetic moments of the iron atoms are tilted with respect to the $c$ axis by angle of $-35.3^{\circ}$. Thus the moments are aligned within a plane (marked with dashed lines) which is lying perpendicular to the direction [111] using the cubic setting. Due to the oxygen deficiency antiferromagnetic coupling occurs between those iron atoms along the $c$ axis, where sequences of alternating units of octahedral $\mathrm{Fe}^{4+} \mathrm{O}_{6}$ and square-pyramidal $\mathrm{Fe}^{3+} \mathrm{O}_{5}$ were found $\left(\mathrm{Fe}^{4+}\right.$ and $\mathrm{Fe}^{3+}$ ions are marked in red and green, respectively).

the alignment of the Fe spins in the two-dimensional network of slightly tilted $\mathrm{Fe} 2 \mathrm{O}_{6}$ octahedra in $\mathrm{Sr}_{8} \mathrm{Fe}_{8} \mathrm{O}_{23}$ is similar to the one within the network of undistorted $\mathrm{FeO}_{6}$ octahedra in $\mathrm{SrFeO}_{3}$, but the ordered moment $\mu_{\text {exp }}=1.69$ (3) $\mu_{\mathrm{B}}$ is considerably smaller. However, the refinement of the helical spin structure resulted in a relatively large residual $R_{\mathrm{M}}=$ 0.168 (defined as $R_{\mathrm{M}}=\sum\left|I_{\mathrm{obs}}-I_{\text {cal }}\right| / \sum I_{\mathrm{obs}}$ ). In particular, the calculated intensity of the reflection $(0.686,2,0.326)_{\mathrm{M}}$ (observed at $2 \theta=29.6^{\circ}$ ) was found to be much smaller than observed, whereas the fit of the other three reflections (given above) was satisfactory.

In order to improve the refinement, the real and imaginary Fourier components of the iron moments were allowed to vary independently. This gave a much better agreement between observed and calculated intensities, resulting in a residual $R_{\mathrm{M}}=0.087$. The refined imaginary component reached a value close to zero. This implies that the magnetic moments of $\mathrm{Sr}_{8} \mathrm{Fe}_{8} \mathrm{O}_{23}$ in the powder sample show a "spin-density wave" (SDW) state (Fig. 10) with an amplitude of 2.54(4) $\mu_{\mathrm{B}}$, considerably smaller than the saturation moments $\mu_{\exp }=$ 2.960(12) $\mu_{\mathrm{B}}$ of the $\mathrm{Fe}^{4+}$ ion in $\mathrm{SrFeO}_{3}$ and 3.55(5) $\mu_{\mathrm{B}}$ of the $\mathrm{Fe}^{3+}$ ion in $\mathrm{Sr}_{4} \mathrm{Fe}_{4} \mathrm{O}_{11}$ (Ref. 8).

\section{Phase IV}

For the refinement of the magnetic structure of phase IV we used the tetragonal setting of $\mathrm{Sr}_{8} \mathrm{Fe}_{8} \mathrm{O}_{23}$, where the propagation vector is $\boldsymbol{k}=\left(0,0, \frac{1}{2}\right)$. Thus the magnetic

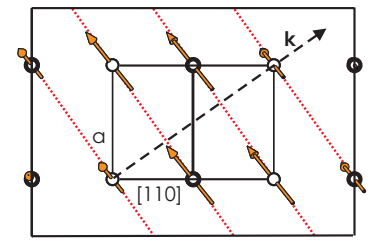

Phase I $\left(\mathrm{SrFeO}_{3}\right)$

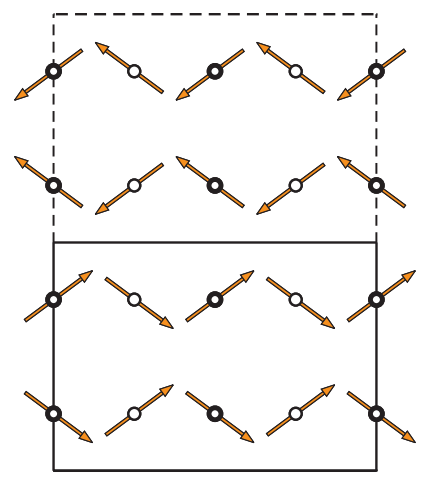

Phase IV

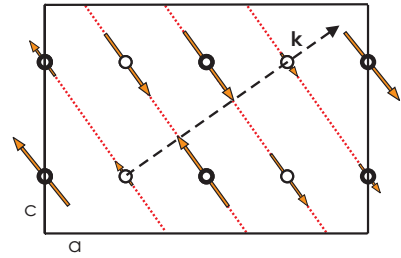

Phase III $\left(\mathrm{Sr}_{8} \mathrm{Fe}_{8} \mathrm{O}_{23}\right)$

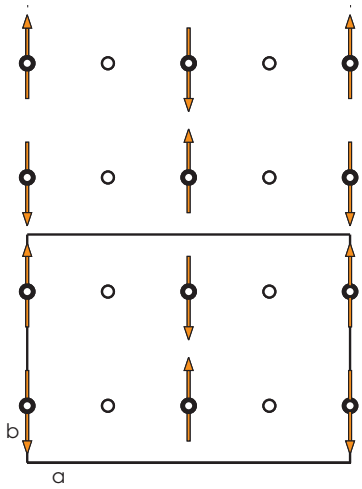

Phase VII $\left(\mathrm{Sr}_{4} \mathrm{Fe}_{4} \mathrm{O}_{11}\right)$
FIG. 11. (Color online) Magnetic ordering of the iron moments in the system $\mathrm{SrFeO}_{3-\delta}$. The magnetic structures are shown as projections along the $b$ and $c$ axes of $\mathrm{Sr}_{8} \mathrm{Fe}_{8} \mathrm{O}_{23}$ and $\mathrm{Sr}_{4} \mathrm{Fe}_{4} \mathrm{O}_{11}$, respectively. For $\mathrm{SrFeO}_{3}$ the cubic unit cell is also shown. The iron moments in $\mathrm{SrFeO}_{3}$ and $\mathrm{Sr}_{8} \mathrm{Fe}_{8} \mathrm{O}_{23}$ show a helical and a sine-wave modulated magnetic ordering with a propagation vector $\boldsymbol{k}$ parallel to the direction [111] of the cubic unit cell, respectively. The canting angle of the moments with respect to the $c$ axis is $\theta=-35.3^{\circ}$. A further decrease of oxygen content leads to commensurate magnetic ordering, where the moments are still canted with respect to the $c$ axis. For this phase the magnetic structure can be described with the propagation vector $\boldsymbol{k}=\left(0,0, \frac{1}{2}\right)$ using the tetragonal setting of $\mathrm{Sr}_{8} \mathrm{Fe}_{8} \mathrm{O}_{23}$. Thus the magnetic structure requires a doubling of the tetragonal $c$ axis. A further reduction of oxygen leads to a simple collinear structure of the $\mathrm{Fe}^{3+}$ sublattice of $\mathrm{Sr}_{4} \mathrm{Fe}_{4} \mathrm{O}_{11}$, whereas the moments of the $\mathrm{Fe}^{4+}$ ions in this phase are not three-dimensionally ordered. ${ }^{8}$

structure requires a doubling of the $c$ axis of the tetragonal unit cell. The strongest magnetic intensity could be observed for the reflection $\left(0,0, \frac{1}{2}\right)_{\text {tetr }}$ at $9.5^{\circ}$ (Fig. 6). This suggests a ferromagnetic ordering of the iron moments (all at $z=$ 0.25 and 0.75 in the unit cell) within the $a b$ plane, and a spin sequence along the $c$ axis,,$++--+\ldots$ First the magnetic structure was refined using a model where the moments are aligned parallel to the $a$ axis. With this model the observed and calculated intensities of the reflection $\left(0,0, \frac{1}{2}\right)_{\mathrm{M}}$ were found to be in good agreement, whereas the calculated intensity of the $\left(0,0,1 \frac{1}{2}\right)_{\text {tetr }}$ (at $2 \theta=28.9^{\circ}$ ) was found to be too low. But it could be seen that the magnetic reflections $\left(2,0, \frac{1}{2}\right)_{M}$ and $\left(0,2, \frac{1}{2}\right)_{M}$ are very close to the position of the reflection $\left(0,0,1 \frac{1}{2}\right)_{\text {tetr. }}$. Therefore we assumed a magnetic ordering where the second magnetic component was aligned parallel to the $c$ axis. Due to the fact that magnetic intensity was only observed for magnetic reflections with $h, k=2 n$ an antiferromagnetic spin sequence,,$+-+-+\ldots$ along the $a$ axis is expected. Then the lattice parameter $a$ of the 
TABLE III. Magnetic phases in the system $\mathrm{SrFeO}_{3-\delta}(0 \leqslant \delta \leqslant 0.25)$ found by single-crystal or powder neutron diffraction, as indicated. In this system the magnetic moments of the iron atoms show commensurate antiferromagnetic (AF), helical, and amplitude-modulated spin-density wave (SDW) structures. Note that not all the observed spin order types could be attributed to the known crystal structures.

\begin{tabular}{|c|c|c|c|c|c|}
\hline Magnetic phase & Propagation vector $\boldsymbol{k}$ & Type of spin order & $T_{\mathrm{N}}(\mathrm{K})$ & Samples & Crystal structure \\
\hline Phase I & $(0.13,0.13,0.13)_{\mathrm{cub}}$ & Helical & 133(1), 134 (Ref. 1) & $\mathrm{SrFeO}_{2.87-3.00}$ (crystal) & $\mathrm{SrFeO}_{3}$ \\
\hline Phase II & $(0.20,0.20,0.20)_{\text {cub }}$ & Helical & $75(2)$ & $\mathrm{SrFeO}_{2.87}$ (crystal) & $\mathrm{Sr}_{8} \mathrm{Fe}_{8} \mathrm{O}_{23}$ \\
\hline Phase III & $(0.17,0.17,0.17)_{\text {cub }}(0.687,0,0.326)_{\text {mon }}$ & SDW & $75(2)$ & $\mathrm{SrFeO}_{2.87}$ (powder) & $\mathrm{Sr}_{8} \mathrm{Fe}_{8} \mathrm{O}_{23}$ \\
\hline Phase IV & $\left(0,0, \frac{1}{4}\right)_{\mathrm{cub}}\left(0,0, \frac{1}{2}\right)_{\mathrm{tetr}}$ & Canted AF & $65(4)$ & $\mathrm{SrFeO}_{2.77-3.00}$ (crystal) & Unknown \\
\hline Phase V & $(0.30,0.30,0.75)_{\mathrm{cub}}$ & SDW or helical & $110(4)$ & $\mathrm{SrFeO}_{2.77}$ (crystal) & Unknown \\
\hline Phase VI & $(0.79,0.79,0)_{\mathrm{cub}}$ & SDW or helical & $60(5)$ & $\mathrm{SrFeO}_{2.77}$ (crystal) & Unknown \\
\hline Phase VII & 0 & $\mathrm{AF}$ & 232(2), 232(4) (Ref. 8) & $\mathrm{SrFeO}_{2.77}$ (crystal) & $\mathrm{Sr}_{4} \mathrm{Fe}_{4} \mathrm{O}_{11}$ \\
\hline
\end{tabular}

magnetic cell is only half of that of the tetragonal unit cell, but identical to the $c$ axis of orthorhombic structure of $\mathrm{Sr}_{4} \mathrm{Fe}_{4} \mathrm{O}_{11}$. With this model, where the moments were allowed to vary in the $a c$ plane, we finally obtained a satisfactory refinement resulting in a residual $R_{\mathrm{M}}=0.067$. It was found that the moments are tilted with respect to the $c$ axis with an angle of $54(1)^{\circ}$, which is considerably larger than the value $35.3^{\circ}$ found for $\mathrm{SrFeO}_{3}$ and $\mathrm{Sr}_{8} \mathrm{Fe}_{8} \mathrm{O}_{23}$ (Fig. 11). In the tetragonal setting one cannot distinguish whether the iron moments are aligned in the $a c$ or the $b c$ plane.

In order to estimate the content of the magnetic phase IV we assumed a magnetic moment of the iron atoms of $\mu_{\exp }=$ $3.0 \mu_{\mathrm{B}}$. The phase analysis of the powder sample with an oxygen deficiency of $\delta=0.13$ showed that the powder contains $78 \%$ phase III, $14 \%$ phase I, and $8 \%$ phase IV. These fractions could be determined with a statistical accuracy of $2 \%$, but are subject to a systematic error arising from the unknown ordered moment of phase IV. As we have seen in Sec. III A, these magnetic phases are not necessarily fully congruent with oxygen vacancy-ordered structures. For the powder sample with $\delta=0.19$ it was found that the sample additionally contains phase VII, which is characteristic of orthorhombic $\mathrm{Sr}_{4} \mathrm{Fe}_{4} \mathrm{O}_{11}$, while phase $\mathrm{I}$ is absent. For this powder sample the phase analysis resulted in the following composition: $45 \%$ phase III, $40 \%$ phase VII, and $15 \%$ phase IV. As discussed in Sec. III A, phase IV may belong to a new ferrate structure, which could not be identified in the present study. In mixed-phase samples, it is quite difficult to determine the crystal structure of minority phases, because the strong reflections of all the different phases overlap, and the superstructure reflections of the minority phases are rather weak and difficult to measure with good accuracy.

\section{CONCLUSIONS}

Our experiments have revealed a surprising variety of magnetic ordering patterns in oxygen-deficient $\mathrm{SrFeO}_{3-\delta}$. Table III lists the basis properties of the seven structures that could be identified, and Fig. 11 depicts those structures for which a refinement could be obtained. This variety is a consequence of a confluence of several factors including competing magnetic interactions, charge fluctuations, and the ramified bond network created by the oxygen vacancy arrays. In order to understand the influence of these factors, it is useful to begin our discussion by considering $\mathrm{BiFeO}_{3}$, an insulating compound with $\mathrm{Fe}^{3+}$ ions that crystallizes in a rhombohedrally distorted, pseudocubic perovskite structure. $\mathrm{BiFeO}_{3}$ exhibits nearly collinear antiferromagnetism with a high Néel temperature of $T_{\mathrm{N}}=643 \mathrm{~K} \cdot{ }^{31}$ (A long-wavelength cycloidal modulation is induced by exchange anisotropies due to the rhombohedral lattice distortion. ${ }^{32}$ ) Its close analog $\mathrm{NdFeO}_{3}$ exhibits an even larger $T_{\mathrm{N}}=760 \mathrm{~K}^{33}$ The high ordering temperatures reflect strong, unfrustrated superexchange interactions in a three-dimensional bond network. The sheets of trivalent $\mathrm{Fe} 2$ atoms in $\mathrm{Sr}_{4} \mathrm{Fe}_{4} \mathrm{O}_{11}$ can be regarded as a two-dimensional segment of this network, cut along the [110] planes of the cubic cell. As a result, Fe 2 spins along the orthorhombic $c$ axis are coupled via $180^{\circ}$ bonds like those in $(\mathrm{Bi}, \mathrm{Nd}) \mathrm{FeO}_{3}$ while those along the $a$ axis are coupled via weaker $90^{\circ}$ bonds. The coupling between the antiferromagnetic $\mathrm{Fe} 2$ atom layers via the intervening Fe1 atom chains is presumably even weaker. This dilution of the exchange bond network is responsible for the much lower ordering temperature $T_{\mathrm{N}}=232 \mathrm{~K}$ of $\mathrm{Sr}_{4} \mathrm{Fe}_{4} \mathrm{O}_{11}$ compared to $\mathrm{BiFeO}_{3}$ and $\mathrm{NdFeO}_{3}$. The resulting amplitude modulation of the ordered moment, which implies paramagnetic Fe1 atom chains even as $T \rightarrow 0$, deserves further investigation.

The incommensurate helical order of $\mathrm{SrFeO}_{3}$, with uniform $\mathrm{Fe}^{4+}$ valence, can be traced to a delicate competition between ferromagnetic double-exchange and antiferromagnetic super-exchange interactions on the verge of a metal-insulator transition. ${ }^{34}$ In fact, its close analog $\mathrm{CaFeO}_{3}$, whose electronic bandwidth is lowered by an orthorhombic tilt distortion of the $\mathrm{FeO}_{6}$ octahedra, undergoes a metal-insulator transition associated with charge disproportionation at $290 \mathrm{~K}$, and a transition to helical magnetism at $T_{\mathrm{N}}=115 \mathrm{~K} .^{25,35-37}$ The helix propagation vector of $\boldsymbol{k}=(0.161,0.161,0.161)_{\text {cub }}$ in the insulating state of $\mathrm{CaFeO}_{3}$ reflects a smaller ratio of double-exchange and super-exchange interactions compared to the one in the metallic state of $\mathrm{SrFeO}_{3}$, where $\boldsymbol{k}=(0.128$, $0.128,0.128)_{\text {cub }}$.

In the range $0<\delta<0.25$ both frustration mechanisms are present and conspire to suppress the magnetic ordering temperatures even further, into the range $60 \mathrm{~K} \leqslant T \leqslant 75 \mathrm{~K}$. The low-temperature-ordered moments follow an analogous trend. Among the resulting magnetic phases II-VI, only phases II and III could be uniquely associated with a vacancy-ordered structure, namely $\mathrm{Sr}_{8} \mathrm{Fe}_{8} \mathrm{O}_{23}$. In this structure, the vacancy order induces segregation of the $\mathrm{Fe}$ valence into different lattice planes. In contrast to $\mathrm{Sr}_{4} \mathrm{Fe}_{4} \mathrm{O}_{11}$, however, the network of exchange bonds between tetravalent $\mathrm{Fe} 1$ and $\mathrm{Fe} 3$ atoms is not as strongly disrupted as in $\mathrm{Sr}_{4} \mathrm{Fe}_{4} \mathrm{O}_{11}$, and the sheets of 
$\mathrm{Fe} 2$ atoms are in the intermediate valence state $\mathrm{Fe}^{3.5+}$ rather than $\mathrm{Fe}^{3+}$. As a result, the magnetic ordering patterns in the monoclinic, insulating state for $T \leqslant T_{\mathrm{S}}=75 \mathrm{~K}$ resemble the helix states with propagation vector along the cubic [111] axis observed in $\mathrm{SrFeO}_{3}$ and $\mathrm{CaFeO}_{3}$. The incommensurability of phase III, $\Delta=0.169$, is larger than the one of $\mathrm{SrFeO}_{3}$ and close to the one of $\mathrm{CaFeO}_{3}$, in accord with the expectation that the strength of the antiferromagnetic superexchange increases relative to the ferromagnetic double exchange in compounds with more localized electrons. In contrast to $\mathrm{SrFeO}_{3}$ and $\mathrm{CaFeO}_{3}$, however, phase III is a collinear, amplitude-modulated state. This aspect is qualitatively analogous to the antiferromagnetic state observed in $\mathrm{Sr}_{4} \mathrm{Fe}_{4} \mathrm{O}_{11}$.

While phase III was observed in a $\mathrm{SrFeO}_{2.87}$ powder sample with $\mathrm{Sr}_{8} \mathrm{Fe}_{8} \mathrm{O}_{23}$ as majority vacancy-ordered phase, we found a different magnetic structure, phase II, in the $\mathrm{Sr}_{8} \mathrm{Fe}_{8} \mathrm{O}_{23}$ volume fraction of a single crystal with the same oxygen stoichiometry. Phase II is a commensurate helix state with $\Delta=0.2$. This indicates a subtle balance between amplitude-modulated and helix states in this range of oxygen deficiencies. Details of the oxygen distribution (such as the density of possible randomly incorporated oxygen defects in $\mathrm{Sr}_{8} \mathrm{Fe}_{8} \mathrm{O}_{23}$ ) or the microstructure of the lattice appear to determine the balance between these nearly degenerate magnetic states.

Finally, we point out that the magnetic microstructure resulting from this delicate phase balance appears to exert a key influence on the magnetotransport properties. A prime example is the commensurate phase IV, which occupies only a very small volume fraction in nominally stoichiometric
$\mathrm{SrFeO}_{3.00}$ single crystals, but nonetheless triggers a large-scale modification of the domain structure of the incommensurate majority phase I. This, in turn, induces a resistivity jump $T=60 \mathrm{~K}$, the onset of magnetic order in phase IV. The magnetic field dependence of the phase-IV ordering temperature is therefore presumably responsible for the large magnetoresistance in $\mathrm{SrFeO}_{3.00}$ crystals. ${ }^{11,12}$

In the future, it will be interesting to explore the influence of chemical substitution (such as $\mathrm{La}$ for $\mathrm{Sr}$ and/or Co for $\mathrm{Fe}$ ), pressure, and magnetic field on the interplay between oxygen vacancy order, magnetic microstructure, and magnetoresistance of $\mathrm{SrFeO}_{3}$ and other ferrates. ${ }^{15,38}$ We also note that a delicate dependence of the magnetic texture on external parameters and associated transport anomalies have been observed in other incommensurately modulated magnets such as $\mathrm{BiFeO}_{3}, \mathrm{MnSi}$, and $\mathrm{MnGe}{ }^{17-19} \mathrm{~A}$ detailed understanding of these phenomena is an interesting target for future research.

\section{ACKNOWLEDGMENTS}

We acknowledge fruitful discussions with T. Arima, D. Efremov, G. Khaliullin, D. Peets, and Y. Tokura, and thank N. Cavadini and D. Sheptyakov for help with the measurements at the Paul-Scherrer-Institut. We thank the German Science Foundation (DFG) for financial support under collaborative Grant No. SFB/TRR 80. This paper is partly based on the results of the experiments carried out at the Swiss spallation neutron source SINQ, Paul-Scherrer-Institut, Villigen, Switzerland. *reehuis@helmholtz-berlin.de

†b.keimer@fkf.mpg.de

${ }^{1}$ T. Takeda, Y. Yamaguchi, and H. Watanabe, J. Phys. Soc. Jpn. 33, 967 (1972).

${ }^{2}$ Y. Takeda, K. Kanno, T. Takada, O. Yamamoto, M. Takano, N. Nakayama, and Y. Bando, J. Solid State Chem. 63, 237 (1986).

${ }^{3}$ M. Takano, T. Okita, N. Nakayama, Y. Bando, Y. Takeda, O. Yamamoto, and J. B. Goodenough, J. Solid State Chem. 73, 140 (1988).

${ }^{4}$ J. Mizusaki, M. Okayasu, S. Yamauchi, and K. Fueki, J. Solid State Chem. 99, 166 (1992).

${ }^{5}$ T. C. Gibb, J. Mater. Chem. 4, 1445 (1994).

${ }^{6}$ J. P. Hodges, S. Short, J. D. Jorgensen, X. Xiong, B. Dabrowski, S. M. Mini, and C. W. Kimball, J. Solid State Chem. 151, 190 (2000).

${ }^{7}$ J. B. MacChesney, R. C. Sherwood, and J. F. Potter, J. Chem. Phys. 43, 1907 (1965).

${ }^{8}$ M. Schmidt, M. Hofmann, and S. J. Campbell, J. Phys.: Condens. Matter 15, 8691 (2003).

${ }^{9}$ H. D'Hondt, A. M. Abakumov, J. Hadermann, A. S. Kalyuzhnaya, M. G. Rozova, E. V. Antipov, and G. Van Tendeloo, Chem. Mater. 20, 7188 (2008).

${ }^{10}$ Y. M. Zhao, R. Mahendiran, N. Nguyen, B. Raveau, and R. H. Yao, Phys. Rev. B 64, 024414 (2001).

${ }^{11}$ A. Lebon, P. Adler, C. Bernhard, A. V. Boris, A. V. Pimenov, A. Maljuk, C. T. Lin, C. Ulrich, and B. Keimer, Phys. Rev. Lett. 92, 037202 (2004).
${ }^{12}$ P. Adler, A. Lebon, V. Damljanović, C. Ulrich, C. Bernhard, A. V. Boris, A. Maljuk, C. T. Lin, and B. Keimer, Phys. Rev. B 73, 094451 (2006).

${ }^{13}$ E. K. Hemery, G. V. M. Williams, and H. J. Trodahl, Phys. Rev. B 75, 092403 (2007).

${ }^{14}$ G. V. M. Williams, E. K. Hemery, and D. McCann, Phys. Rev. B 79, 024412 (2009).

${ }^{15}$ L. Kienle, P. Adler, J. Strempfer, B. Keimer, V. Duppel, and F. Phillipp, J. Phys. Chem. Solids 68, 73 (2007).

${ }^{16}$ S. Ishiwata, M. Tokunaga, Y. Kaneko, D. Okuyama, Y. Tokunaga, S. Wakimoto, K. Kakurai, T. Arima, Y. Taguchi, and Y. Tokura, Phys. Rev. B 84, 054427 (2011).

${ }^{17}$ M. Ramazanoglu, W. Ratcliff II, H. T. Yi, A. A. Sirenko, S.-W. Cheong, and V. Kiryukhin, Phys. Rev. Lett. 107, 067203 (2011).

${ }^{18}$ C. Pfleiderer, T. Adams, A. Bauer, W. Biberacher, B. Binz, F. Birkelbach, P. Böni, C. Franz, R. Georgii, M. Janoschek, F. Jonietz, T. Keller, R. Ritz, S. Mühlbauer, W. Münzer, A. Neubauer, B. Pedersen, and A. Rosch, J. Phys.: Condens. Matter 22, 164207 (2010).

${ }^{19}$ N. Kanazawa, Y. Onose, T. Arima, D. Okuyama, K. Ohoyama, S. Wakimoto, K. Kakurai, S. Ishiwata, and Y. Tokura, Phys. Rev. Lett. 106, 156603 (2011).

${ }^{20}$ A. Maljuk, A. Lebon, V. Damljanović, C. Ulrich, C. T. Lin, P. Adler, and B. Keimer, J. Cryst. Growth 291, 412 (2006).

${ }^{21}$ A. Maljuk, J. Strempfer, C. Ulrich, A. Lebon, and C. T. Lin, J. Cryst. Growth 257, 427 (2003). 
${ }^{22}$ J. Rodríguez-Carvajal, FULLPROF: A program for Rietveld refinement and pattern matching analysis, Abstracts of the Satellite Meeting on Powder Diffraction of the XV Congress of the IUCr, Toulouse, France, 1990, p. 127.

${ }^{23}$ V. F. Sears, in International Tables of Crystallography, edited by A. J. C. Wilson, Vol. C (Kluwer, Dordrecht, 1992), p. 383.

${ }^{24} \mathrm{P}$. J. Brown, in International Tables of Crystallography, edited by A. J. C. Wilson, Vol. C (Kluwer, Dordrecht, 1992), p. 391.

${ }^{25}$ P. M. Woodward, D. E. Cox, E. Moshopoulou, A. W. Sleight, and S. Morimoto, Phys. Rev. B 62, 844 (2000).

${ }^{26}$ R. D. Shannon, Acta Crystallogr. A 32, 751 (1976).

${ }^{27}$ J. Herrero-Martin, G. Subias, J. Garcia, J. Blasco, and M. Concepción Sánchez, Phys. Rev. B 79, 045121 (2009).

${ }^{28}$ D. S. Inosov, D. V. Evtushinsky, V. B. Zabolotnyy, A. A. Kordyuk, B. Büchner, R. Follath, H. Berger, and S. V. Borisenko, Phys. Rev. B 79, 125112 (2009).
${ }^{29} \mathrm{Ph}$. Leininger, D. Chernyshov, A. Bosak, H. Berger, and D. S. Inosov, Phys. Rev. B 83, 233101 (2011).

${ }^{30}$ E. F. Bertaut, Acta Crystallogr., Sect. A 24, 217 (1968); J. Phys., Colloq. C1 32, 462 (1971); J. Magn. Magn. Mater. 24, 267 (1981).

${ }^{31}$ I. Sosnowska, R. Przeniosło, P. Fischer, and V. A. Murashov, J. Magn. Magn. Mater. 160, 384 (1996).

${ }^{32}$ F. Kubel and H. Schmid, Acta Crystallogr., Sect. B 46, 698 (1990).

${ }^{33}$ R. Przeniosło, I. Sosnowska, and P. Fischer, J. Magn. Magn. Mater. 140-144, 2153 (1995).

${ }^{34}$ C. Ulrich, D. Efremov, G. Khaliullin, and B. Keimer (unpublished).

${ }^{35}$ Y. Takeda, S. Naka, M. Takano, T. Shinjo, T. Takeda, and M. Shimada, Mater. Res. Bull. 13, 61 (1978).

${ }^{36}$ M. Takano, S. Nasu, T. Abe, K. Yamamoto, S. Endo, Y. Takeda, and J. B. Goodenough, Phys. Rev. Lett. 67, 3267 (1991).

${ }^{37}$ S. Kawasaki, M. Takano, R. Kanno, T. Takeda, and A. Fujimori, J. Phys. Soc. Jpn. 67, 1529 (1998).

${ }^{38}$ P. Adler and S. Ghosh, Solid State Sci. 5, 445 (2003). 